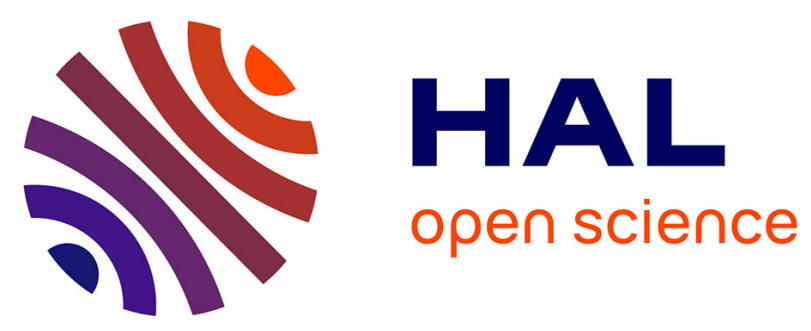

\title{
Comportements et pratiques des médecins : exercer dans les zones les moins dotées, cela fait-il une différence ?
}

\author{
Julien Silhol, Bruno Ventelou, Anna Zaytseva, Claire Marbot
}

\section{To cite this version:}

Julien Silhol, Bruno Ventelou, Anna Zaytseva, Claire Marbot. Comportements et pratiques des médecins : exercer dans les zones les moins dotées, cela fait-il une différence ?. Revue française des affaires sociales, 2019, 1 (2), pp.213-249. 10.3917/rfas.192.0213 . hal-02475133

\section{HAL Id: hal-02475133 \\ https://hal-amu.archives-ouvertes.fr/hal-02475133}

Submitted on 17 Feb 2020

HAL is a multi-disciplinary open access archive for the deposit and dissemination of scientific research documents, whether they are published or not. The documents may come from teaching and research institutions in France or abroad, or from public or private research centers.
L'archive ouverte pluridisciplinaire HAL, est destinée au dépôt et à la diffusion de documents scientifiques de niveau recherche, publiés ou non, émanant des établissements d'enseignement et de recherche français ou étrangers, des laboratoires publics ou privés. 


\title{
Comportements et pratiques des médecins : exercer dans les zones les moins dotées, cela fait-il une différence?
}

\author{
Claire MARBOT, Julien SILHOL, Bruno VENTELOU, Anna ZAYTSEVA
}

\section{Résumé}

Selon les projections récentes, les effectifs de médecins libéraux diminueront de $30 \%$ d'ici à 2027 et la densité standardisée diminuerait jusqu'en 2023, créant des poches de sous-densité relativement nombreuses sur le territoire français métropolitain. L'article s'intéresse aux ajustements que les médecins généralistes de ville mettent en œuvre lorsque, sur leur territoire, ils sont d'ores et déjà confrontés à cette raréfaction. Les données utilisées sont celles du troisième panel des médecins généralistes enrichies d'indicateurs fournies par la CNAMTS. Nous nous sommes appuyés sur l'indicateur d'accessibilité potentielle localisé, développé par l'IRDES et la DREES pour définir les zones les moins dotées en généralistes. En comparant les comportements des généralistes exerçant dans les zones les moins dotées à leurs homologues des zones mieux dotées, il est apparu d'abord que le planning d'activité du médecin s'intensifie : nos données semblent en effet montrer que les rythmes de consultation dans les zones les moins dotées sont plus élevés, alors que le temps de travail global des généralistes s'avère peu réactif à la densité en médecins alentour. On note aussi quelques différences statistiquement significatives sur les pratiques médicales : usage de certains médicaments, renvoi vers des soins paramédicaux, suivis gynécologique probablement un peu moins réguliers, etc. Cependant, il semble que les différences ne sont pas statistiquement significatives pour les indicateurs de qualité des pratiques rattachés au dispositif de rémunération sur objectifs de santé publique (ROSP).

\section{Introduction}

La densité médicale en France est, pour le moment, l'une des plus importantes des pays de l'Organisation de la coopération et du développement économique (OECD, 2016). Mais, selon les projections récentes, sous l'hypothèse de prolongement des comportements et de la législation actuelle, les effectifs de médecins libéraux ${ }^{1}$ diminueraient jusqu'en 2027 et seraient à cette date inférieurs de $24 \%$ à leur niveau de 2012 (Bachelet, 2017). C'est la conséquence des départs en retraite de classes d'âge nombreuses de médecins formés avant l'application d'un numerus clausus très restreint à partir du milieu des années 1970. Cette baisse, accompagnée d'une croissance de la population française, se traduira mécaniquement par une densité médicale plus faible au niveau national. 
En outre, au-delà d'une logique purement quantitative, le système français de délivrance des soins fait actuellement face à trois problèmes. Tout d'abord, la demande de soins ne cesse de croître, du fait à la fois du vieillissement de la population et de l'augmentation de la part des patients souffrant de maladies chroniques (Dormont, 2012). Ensuite, on assiste à une baisse de l'offre de travail moyenne des médecins libéraux, liée aux aspirations des jeunes générations et au nombre croissant de médecins cumulant emploi et retraite, dont le temps de travail est souvent plus faible. Le troisième problème est celui des inégalités géographiques d'installation (Vergier, 2016). La répartition spatiale des médecins généralistes (MG) est moins inégalitaire que ce qui est observé pour les autres professions de santé (Vergier et Chaput, 2017), mais une baisse marquée du choix d'installation en libéral parmi les nouvelles générations de médecins pourrait augmenter le risque de "tensions » sur de nombreux territoires. Ainsi, dans les communes isolées, $52 \%$ de l'accessibilité aux MG repose sur des professionnels de plus de 55 ans, susceptibles de cesser leur activité dans les années à venir (Vergier et Chaput, 2017).

Cette rareté relative de la ressource humaine en soins primaires pourrait poser des problèmes aux populations, en termes de facilité d'accès aux soins, de délais d'attente (Millien, 2018), voire de qualité de vie en santé. Du côté des médecins eux-mêmes, l'exercice dans les zones les moins dotées nécessite certainement des ajustements, ne serait-ce que pour mieux répondre aux besoins et difficultés particulières vécues par leurs patients sur le territoire.

De fait, en raison de l'inégale répartition des médecins sur le territoire, certaines configurations de sous-densité existent dès maintenant et pourraient être considérées comme une situation avancée de celle qui pourrait se produire plus tard sur des portions plus larges de la France. Nous proposerons donc une étude des cas de sous-densité médicale déjà observés et de leurs liens possibles avec des comportements ou des pratiques médicales spécifiques. Nous utilisons pour ce faire les données du troisième Panel d'observation des pratiques et des conditions d'exercice en médecine générale (Panel), qui offre une série d'informations pour une population représentative de MG français, y compris ceux en situation de sous-densité (Le Maréchal, 2016).

L'étude propose d'abord une méthodologie pour repérer les zones de tensions et identifier les MG du Panel qui exercent déjà dans des territoires caractérisés par des pénuries d'offre de soins. Nous proposerons ensuite une étude des liens entre les situations de sous-densité médicale observées et les volumes d'activité des MG sur la semaine (une semaine ordinaire), y compris leurs temps dédiés aux patients et les rythmes de consultation mis en place. Cette question des rythmes de pratique rejoindra en fait, assez naturellement, celle de la qualité des soins. Une littérature relativement abondante, mais pas toujours conclusive, met en effet en lumière les connections possibles entre l'accélération des rythmes de pratiques et la qualité de soins offerts par les médecins de soins primaires (Zyzanski, 1998 ; Holmboe, 2006 ; Hutton, 2007 ; Freeman, 2002 ; Ventelou, 2010 ; Videau, 2010). Nous nous intéresserons donc plus finement à l'adaptation des pratiques des MG confrontés à des situations de zones parmi les moins dotées, pour détecter d'éventuels effets sur la qualité des prises en charge ; les marqueurs de qualité de pratiques retenus reposeront notamment sur la Rémunération sur Objectifs de Santé Publique (ROSP) et, pour dépasser cet indicateur de « qualité des pratiques » probablement biaisé par son inscription en tant que base de rémunération, sur des éléments de la vague 2 du troisième Panel, qui interrogeait les MG sur leur prise en charge des soins gynécologiques. 


\section{Matériel et méthodes}

\subsection{Données}

Les analyses présentées dans cet article ont été réalisées à partir de l'échantillon national du panel d'observation des pratiques et des conditions d'exercice en médecine générale. La troisième édition du Panel - utilisée ici - a été collectée entre 2014 et 2017 grâce à un partenariat entre la Direction de la recherche, des études, de l'évaluation et des statistiques (DREES), les observatoires régionaux de la santé (ORS) et les unions régionales des professionnels de santé médecins libéraux (URPS-ML) de Poitou-Charentes, Pays de la Loire et Provence-Alpes-Côte d'Azur.

L'échantillon a été obtenu à partir du Répertoire partagé des professionnels de santé (RPPS) par tirage aléatoire stratifié sur le sexe, l'âge, le volume d'activité et la densité médicale (Verger, 2017). Ce panel est représentatif de la population des MG du territoire français métropolitain, hormis ceux pratiquant des modes d'exercice particuliers de façon exclusive ou ayant des projets de cessation d'activité ou de déménagement dans l'année. Sur les 3839 MG éligibles contactés par téléphone lors de la vague d'inclusion, 1590 MG (41\%) ont au départ accepté de participer au Panel, et 948 participaient toujours à la cinquième et dernière vague d'enquête au printemps 2017. Parmi les 5 vagues d'enquête (passées essentiellement par téléphone, sauf quelques questionnaires remplis sur internet), deux sont utilisées ici ; la vague d'inclusion, au cours de laquelle des questions ont été posées au MG sur son temps de travail et son cabinet, la deuxième vague, relative à l'implication des MG dans les suivis gynécologiques et de grossesses.

Les enquêtes du Panel sont enrichies de données sur les caractéristiques professionnelles du MG (région d'exercice, nature de l'exercice, mode de conventionnement) et de données administratives de l'Assurance maladie : nombre annuel d'actes, caractéristiques de la patientèle et données de prescriptions (données issues des relevés individuels d'activité et de prescription - RIAP) ainsi que des indicateurs de qualité des pratiques (nombre de points obtenus à différents indicateurs de la ROSP.

Une pondération spécifique a été calculée pour effectuer les analyses. La distinction entre MG des zones les moins dotées et les autres étant centrale dans cette étude, cette pondération spécifique a pour but d'assurer la représentativité des MG du Panel situés dans ce type de zone (et de manière symétrique d'assurer la représentativité des MG du Panel en dehors de ces zones). Trois dimensions ont été retenues pour rendre le Panel représentatif : le sexe, l'âge en trois tranches (les seuils étant 50 et 58 ans au moment de l'inclusion) et le niveau d'activité, en trois tranches aussi, dont les seuils correspondent au premier et au troisième quartiles. 


\subsection{Définition des zones les moins dotées}

\subsubsection{L'indicateur d'accessibilité potentielle aux soins (APL)}

Dans les études, deux indicateurs d'accessibilité spatiale aux MG sont traditionnellement utilisés : d'une part, la densité en MG dans un territoire et, d'autre part, la distance d'accès, pour une population donnée, au médecin le plus proche. Ces indicateurs présentent l'intérêt d'être à la fois facilement calculables et très intuitifs pour les citoyens et les décideurs. Toutefois, ils présentent des limites importantes (Vergier et Chaput, 2017). En effet, la densité nécessite implicitement de supposer que les patients d'un territoire ne consultent pas au-delà des frontières qui le délimitent. Par ailleurs, la distance d'accès au cabinet le plus proche est un indicateur qui s'affranchit des frontières, mais il ne tient pas compte de la tension entre l'offre et la demande (i.e. du nombre de praticiens accessibles en regard du nombre de patients demandeurs de soins), et donc de la possibilité que les cabinets accessibles soient saturés.

La DREES et l'Institut de recherche et de documentation en économie de la santé (IRDES) ont développé en 2012 un indicateur qui pallie ces déficiences en tenant compte à la fois de la proximité et de la disponibilité des médecins. Il s'agit de l'Accessibilité potentielle localisée (APL) qui présente les avantages de pouvoir se calculer à un niveau fin (la commune et, le cas échéant, l'arrondissement municipal) et, surtout, d'intégrer l'offre et la demande en consultations de MG des communes environnantes (Annexe 1). Il s'affranchit des problèmes relatifs aux frontières tout en prenant en compte les distances d'accès aux cabinets de proximité. Ainsi, l'indicateur d'APL permet, plus finement que les indicateurs classiques, d'identifier les points du territoire où existent des tensions entre offre et besoins de soins.

L'APL ici pris en compte est celui qui a été élaboré selon des paramètres concertés dans le cadre de la définition des zonages qui déterminent les zones d'éligibilité aux aides à l'installation et au maintien des médecins. Il présente notamment l'avantage de ne prendre en compte l'activité des médecins qu'au-delà d'un certain seuil (3600 actes) et en-dessous d'une certaine limite (6000 actes).

\subsubsection{Seuil de définition des zones les moins dotées}

Dans le débat public, le terme de « déserts médicaux » est utilisé pour qualifier les territoires où l'offre de soins est insuffisante par rapport aux besoins de la population. Mais ce terme est imprécis. Il n'existe pas, ni internationalement, ni nationalement, de seuil consensuel audessous duquel un territoire pourrait être classé en « désert médical » : la définition d'un tel seuil est par définition arbitraire. Nous choisissons ici d'adopter une définition relative, fondée sur la distribution des niveaux d'APL (l'indicateur étant calculé pour l'année 2015, avec des paramètres qui sont détaillés en annexe 1) sur le territoire français. Un MG est considéré comme exerçant dans les zones les moins dotées s'il fait partie des $10 \%$ des MG dont l'indicateur d'APL de la commune d'installation est le plus bas. Ainsi, nous identifions les $10 \%$ de MG exerçant dans les zones les moins denses. Mais comme les zones les moins denses regroupent une faible concentration de $\mathrm{MG}$ par habitant, la proportion de la population qui y habite excède largement les $10 \%$. 


\section{Graphique 2.1. Définition des zones les moins dotées}

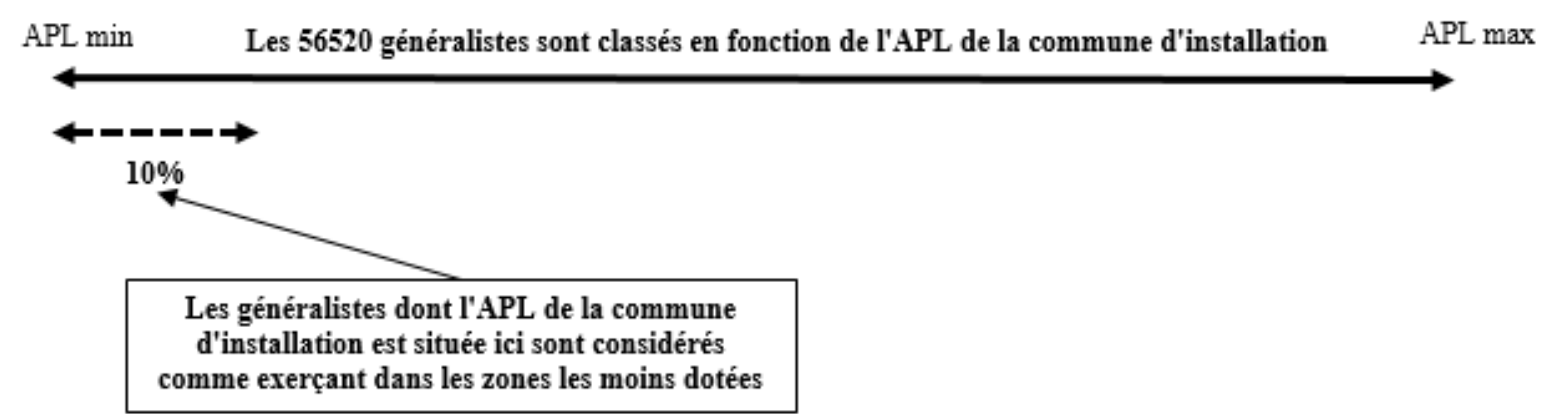

Champ • Généralistes dans le champ du Panel 3 (les médecins exclusivement salariés, à mode d'exercice particulier, ayant des projets de cessation d'activité ou de déménagement dans l'année sont exclus).

Source • Base de sondage du Panel 3, Répertoire partagé de professionnels de santé (RPPS), 2012.

Note $\bullet$ La version de l'APL retenue est celle de 2015.

En pratique, le choix de ce seuil à $10 \%$ correspond à un classement parmi les zones les moins dotées des communes dont l'indicateur d'APL prend des valeurs inférieures à 2,9. Cet ensemble de communes sera donc relativement proche de l'ensemble retenu pour faire partie des zones d'interventions prioritaires (ZIP) vers lesquelles les aides à l'installation et au maintien des généralistes seront fléchées. En effet, le décret ministériel du 13 Novembre 2017 prévoit un classement automatique en ZIP de toutes les communes dont l'indicateur d'APL est inférieur à 2,5, les ARS pouvant ensuite, au cas par cas et en fonction de diagnostics territoriaux plus fins, classer en ZIP des communes dont l'indicateur d'APL est compris entre 2,5 et 4.

\subsubsection{Qui sont les médecins des zones les moins dotées?}

Les MG des zones les moins dotées sont en moyenne un peu plus âgés que leurs confrères (tableau 2.1). Ils n'exercent pas majoritairement dans des communes rurales ${ }^{2}$, cependant, ils y exercent significativement plus que leurs confrères : c'est le cas de 35,2\% des MG des zones les moins dotées, contre $11,7 \%$ des MG exerçant dans les autres zones. La répartition entre hommes et femmes dans les zones les moins dotées est très proche de celle des autres zones.

Les MG des zones les moins dotées sont plus souvent que leurs confrères installés dans des communes où le revenu médian par habitant est élevé. Pratiquement la moitié des MG des zones les moins dotées travaillent au sein d'un cabinet de groupe (c'est-à-dire avec au moins un autre professionnel de santé) ou dans une maison de santé pluridisciplinaire (MSP). La proportion est significativement plus élevée $(56,5 \%)$ pour les MG qui ne font pas partie de des zones les moins dotées. au sens de l'INSEE, en 2010. 
Tableau 2.1. Caractéristiques des médecins généralistes des zones les moins dotées et des autres zones.

\begin{tabular}{|c|c|c|c|c|c|c|c|}
\hline & Hommes & $\begin{array}{l}\text { Moyenne } \\
\text { d'âge }\end{array}$ & $\begin{array}{l}\text { Proportion } \\
\text { ayant plus de } \\
59 \text { ans }\end{array}$ & $\begin{array}{l}\text { Installé dans une } \\
\text { commune rurale }\end{array}$ & $\begin{array}{c}\text { Proportion exerçant } \\
\text { dans les communes } \\
\text { dont le revenu } \\
\text { médian fait partie des } \\
25 \% \text { les plus bas } \\
\end{array}$ & $\begin{array}{c}\text { Proportion exerçant } \\
\text { dans les communes } \\
\text { dont le revenu } \\
\text { médian fait partie des } \\
25 \% \text { les plus hauts } \\
\end{array}$ & $\begin{array}{c}\text { En cabinet de } \\
\text { groupe ou en } \\
\text { MSP }\end{array}$ \\
\hline Zones les moins dotées & $70,6 \%$ & 52,7 ans & $25,5 \%$ & $35,2 \%$ & $21,1 \%$ & $33,7 \%$ & $47,6 \%$ \\
\hline Autres zones & $69,3 \%$ & 52,0 ans & $22,9 \%$ & $11,7 \%$ & $28,9 \%$ & $21,1 \%$ & $56,5 \%$ \\
\hline $\mathrm{p}$-valeur & 0,04 & $<0,01$ & $<0,01$ & $<0,01$ & $<0,01$ & $<0,01$ & $\mathbf{0 , 0 3}$ \\
\hline
\end{tabular}

Champs - Six premières colonnes : généralistes libéraux exerçant en France Métropolitaine en 2010 (les médecins exclusivement salariés, à mode d'exercice particulier, ayant des projets de cessation d'activité ou de déménagement dans l'année sont exclus), soit le champ du panel des MG (56 520 médecins). Dernière colonne : vague d'inclusion au troisième panel des médecins généralistes. Les colonnes 2 et 3 sont obtenues à partir de l'âge des sujets au 1er Janvier 2012.

Sources • DREES, Répertoire Partagé des Professionnels de Santé (RPPS) ; DREES, Unions régionales des professionnels de santé-médecins libéraux (URPS-ML) et Observatoires régionaux de la santé (ORS) PACA, Pays de la Loire et PoitouCharentes, panel d'observation des pratiques et des conditions d'exercice en médecine générale.

Lecture • 70,6 \% des MG travaillant dans les zones les moins dotées sont des hommes.

Note $\bullet$ la p-valeur donnée est celle d'un test de Student pour la deuxième colonne et d'un test du chi2 pour les 6 autres colonnes.

\subsubsection{Variables de contrôle}

Du tableau 2.1, on peut retenir que les MG des zones les moins dotées - et leurs patients présentent des différences sur quelques variables connues pour jouer en elles-mêmes sur les pratiques médicales. Nous avons voulu corriger de ces effets de structure en contrôlant nos analyses, afin d'identifier autant que possible l'effet statistique «toutes choses égales par ailleurs » de l'exercice médical dans les zones les moins dotées. Dans l'ensemble des analyses qui vont suivre, nous utilisons des régressions économétriques qui permettent de prendre en compte les effets de l'âge et du sexe du médecin ainsi que les deux modalités de fonctionnement du cabinet (conventionnement, exercice en groupe). À Paris et dans le département des Hautsde-Seine, on rencontre une concentration exceptionnelle de spécialistes (exerçant en libéral). Aussi nous avons contrôlé l'effet de la densité médicale sur le temps de travail de la localisation du cabinet dans ces zones. Certaines caractéristiques de la patientèle peuvent aussi avoir une influence. Nous prenons en compte le niveau de vie de la commune (évalué par le revenu médian des habitants), la structure par âge des patients et la proportion de patients bénéficiant de la Couverture maladie universelle (CMU). L'utilisation de la variable CMU, outre sa dimension financement des soins, est un indicateur de la précarité sociale au niveau précis de la patientèle du médecin, et s'ajoute à la variable 'revenu médian' de la commune, qui, elle, décrit plus largement l'environnement d'exercice. 


\section{Activité des médecins dans les zones les moins dotées}

\subsection{Zones les moins dotées : davantage d'actes pour un temps de travail comparable}

Dans cette partie, nous cherchons à comparer le nombre moyen d'actes réalisés par les MG des zones les moins dotées à celui réalisé par leurs confrères, ainsi que les temps de travail déclarés de ces deux populations de MG.

Tableau 3.1. Nombre d'actes et temps de travail déclarés en fonction de la densité médicale.

\begin{tabular}{|c|c|c|c|}
\hline & $\begin{array}{c}\text { Nombre d'actes } \\
\text { (1) }\end{array}$ & $\begin{array}{c}\text { Temps de travail } \\
\text { (total) } \\
(2)\end{array}$ & $\begin{array}{c}\text { Temps de travail } \\
\text { (activité libérale) } \\
\text { (3) }\end{array}$ \\
\hline Zones les moins dotées & 5260 & 55,1 & 47,5 \\
\hline Autres zones & 4913 & 54,8 & 47,9 \\
\hline $\begin{array}{l}\text { Variation en pourcentage (zones les moins dotées / } \\
\text { autres zones) }\end{array}$ & $+7,1 \%$ & $+0,5 \%$ & $-0,8 \%$ \\
\hline p-valeur (test de Student) & 0,09 & 0,80 & 0,70 \\
\hline
\end{tabular}

Champ • Généralistes ayant accepté la communication de leur relevé individuel d'activité et de prescription (RIAP), soit 1533 médecins, France métropolitaine.

Source - DREES, URPS-ML et ORS PACA, Pays de la Loire et Poitou-Charentes, panel d'observation des pratiques et des conditions d'exercice en médecine générale, mars 2014.

Notes •

Colonne (1) : Le nombre d'actes est issu des données de l'assurance maladie enrichissant les données de l'enquête. Il correspond à la somme des visites et des consultations effectuées par les médecins en 2013.

Colonne (2) : la question était posée comme suit : «Au final, la semaine dernière/ au cours d'une semaine ordinaire, en comptant toutes vos activités professionnelles, pendant combien d'heures travaillez-vous ? »;

Colonne (3) : la question était «Au final, la semaine dernière/ au cours d'une semaine ordinaire, en comptant toutes vos activités professionnelles, combien d'heures consacrez-vous pour votre activité de médecin libéral de cabinet en ville, y compris les diverses tâches administratives ?»

Les MG exerçants dans les zones les moins dotées réalisent davantage d'actes que les autres $(+7 \% \text {, cette différence étant significative au seuil de } 10 \% \text {, tableau 3.1 })^{3}$. Pour autant, ils ne déclarent pas travailler significativement plus longtemps ${ }^{4}$. Ce résultat nous a paru surprenant et nous a semblé mériter plusieurs analyses complémentaires pour les valider.

Des régressions ont d'abord été estimées, afin de tenir compte des différences structurelles. Il apparaît que, «toutes choses égales par ailleurs », l'écart de temps de travail entre les deux groupes de MG reste non-significatif (Tableau 3.2, nous reprenons les variables de contrôle déjà annoncées à la section 2.2.3). Nous avons également testé la robustesse de ces résultats à des variations du seuil permettant de séparer les zones les moins dotées. De même, la vague d'inclusion du Panel interroge les MG sur certaines activités susceptibles d'impacter le temps de travail dédié à l'activité libérale (temps consacré à des tâches administratives, formation

3 Les résultats présentés dans cette partie sont issus de la sous-population des répondants au troisième panel d'observation des pratiques et condition d'exercice en médecine générale ne s'étant pas opposé à la communication de leur Relevé Individuel d'Activité et de Prescription (RIAP), soit 1553 MG sur 1712.

$4 \quad$ Nous ne voyons pas de raison objective pour laquelle les MG de l'un des deux groupes auraient, plus que les autres, tendance à sous-estimer ou surestimer leurs temps de travail. Aussi, quoique déclarative, cette donnée nous semble fiable pour effectuer une comparaison entre les deux groupes. 
continue, encadrement d'étudiants) ; il est ainsi possible d'obtenir des proxys du temps dédié aux consultations et de comparer leurs moyennes entre les deux types de zone ${ }^{5}$. Les écarts de temps que nous obtenons, quelles que soient les hypothèses faites, restent toujours nonsignificatifs (annexe 2).

Enfin nous avons aussi interrogé notre méthodologie d'approche des zones moins dotées. Celles-ci sont définies à partir de l'indicateur d'APL. Or, l'indicateur prend en compte le nombre d'actes réalisés par les médecins (cf. annexe 1). Aussi, la qualification d'une zone comme faisant partie des moins dotées à l'aide d'un tel indicateur dépend du volume de travail des médecins de la zone. Même si l'indicateur d'APL ici utilisé ne prend l'activité que dans une certaine limite, cela pourrait poser problème lorsqu'il s'agit d'étudier, à rebours, l'influence de la sous-densité sur le temps de travail ou le nombre d'actes réalisés par les médecins : on pourrait assister à une forme de circularité (APL et temps de travail sont liés « par définition »). C'est pourquoi les résultats présentés avec l'indicateur d'APL classique ${ }^{6}$ ont aussi été calculés en utilisant une version de l'indicateur dans laquelle chaque médecin est pris en compte à hauteur d'un équivalent temps plein, et non pas d'une manière relative à son niveau d'activité. Cet indicateur alternatif conduit à des résultats tout à fait proches ${ }^{7}$ (cf. annexe 2).

À partir de nos données, nous venons donc d'établir, d'une manière robuste, que les MG des zones les moins dotées ne travaillent pas davantage que leurs confrères. Le temps de travail des MG serait donc peu élastique aux variations de la densité médicale. Ce résultat questionne. Peut-être qu'une large part du territoire est déjà dans une situation où la demande en soins est suffisante pour permettre aux MG de fixer eux-mêmes leur temps de travail au niveau qui leur convient, sans qu'il ne soit alors possible de détecter d'effet spatial propre aux zones les moins denses.

$5 \quad$ Il se pourrait en effet que des modalités particulières d'organisation dans les zones les moins dotées conduisent à des temps alloués aux consultations plus élevés, pour des temps globaux dévolus à l'activité libérale identiques.

$6 \quad$ Dans sa version de 2015.

$7 \quad$ Nous attribuons cette proximité des résultats au fait que le niveau d'activité des médecins dans le calcul de l'APL est assez largement écrêté : les médecins réalisant entre 3600 et 6000 actes dans l'année sont comptabilisés en fonction de leur niveau d'activité, tandis que ceux réalisant moins de 3600 actes ou plus de 6000 actes ont un niveau d'activité ramené respectivement à 3600 et 6000 actes. 
Tableau 3.2. Nombre d'actes et temps de travail, régressions linéaires multiples.

\begin{tabular}{|c|c|c|c|c|c|c|}
\hline & \multicolumn{2}{|c|}{$\begin{array}{l}\text { Nombre d'actes } \\
\qquad \mathbf{N}=\mathbf{1 5 3 7} \\
\end{array}$} & \multicolumn{2}{|c|}{$\begin{array}{c}\text { Temps de travail } \\
\text { (total) } \\
\mathrm{N}=1491\end{array}$} & \multicolumn{2}{|c|}{$\begin{array}{l}\text { Temps de travail } \\
\text { (activité libérale) } \\
\qquad \mathrm{N}=\mathbf{1 5 0 3}\end{array}$} \\
\hline & Valeur & $\mathbf{p}$ & Valeur & $\mathbf{p}$ & Valeur & $\mathbf{p}$ \\
\hline Constante & 4835 & $<0,01$ & 53,60 & $<0,01$ & 49,60 & $<0,01$ \\
\hline Zones les moins dotées & 336 & $\mathbf{0 , 0 5}$ & $-0,54$ & 0,63 & $-1,19$ & 0,25 \\
\hline \multicolumn{7}{|l|}{ Age } \\
\hline$<50$ ans & -631 & $<0,01$ & $-3,73$ & $<0,01$ & $-2,17$ & $<0,01$ \\
\hline$>58$ ans & -207 & 0,13 & $-3,45$ & $<0,01$ & $-2,33$ & $<0,01$ \\
\hline Femme & -1361 & $<0,01$ & $-7,30$ & $<0,01$ & $-6,25$ & $<0,01$ \\
\hline Secteur 2 ou non conventionné & -1577 & $<0,01$ & $-1,79$ & 0,14 & $-1,21$ & 0,29 \\
\hline Exercice en groupe / MSP & 264 & $\mathbf{0 , 0 2}$ & $-0,75$ & 0,27 & $-0,81$ & 0,20 \\
\hline Zone rurale & 28 & 0,86 & 0,88 & 0,36 & 1,19 & 0,18 \\
\hline Paris et Hauts-de-Seine & 364 & 0,18 & 1,76 & 0,27 & 1,53 & 0,31 \\
\hline \multicolumn{7}{|l|}{ Niveau de vie de la commune } \\
\hline Q1 & 254 & 0,07 & 1,23 & 0,14 & 0,96 & 0,21 \\
\hline Q4 & -336 & $\mathbf{0 , 0 2}$ & 0,00 & 0,99 & $-0,71$ & 0,37 \\
\hline \multicolumn{7}{|l|}{ Caractéristiques de la patientèle } \\
\hline Parts des patients $<16$ ans & 5438 & $<0,01$ & 14,53 & $\mathbf{0 , 0 3}$ & 5,68 & 0,36 \\
\hline Part de patients $\geq 60$ ans & -2354 & $<0,01$ & 12,26 & $<0,01$ & 3,96 & 0,36 \\
\hline Parts des patients CMU & 2696 & $<0,01$ & $-1,05$ & 0,84 & $-2,06$ & 0,67 \\
\hline $\mathrm{R}^{2}$ & \multicolumn{2}{|c|}{0,21} & \multicolumn{2}{|c|}{0,10} & \multicolumn{2}{|c|}{$\mathbf{0 , 0 8}$} \\
\hline
\end{tabular}

Champ • Généralistes ayant accepté la communication de leur RIAP, soit 1533 médecins sur 1712 répondants, France métropolitaine.

Source - DREES, URPS-ML et ORS PACA, Pays de la Loire et Poitou-Charentes, panel d'observation des pratiques et des conditions d'exercice en médecine générale, avril-juillet 2014

\subsection{Le rythme des consultations et le temps de formation comme variables d'ajustement?}

Confrontés à plus d'actes, mais sans offrir plus en termes de plages horaires de consultations, les MG des zones les moins dotées doivent bien trouver des marges d'ajustement. Une hypothèse qui vient naturellement serait d'envisager des variations dans le cadencement des activités de consultations. Ainsi, un indicateur de rythme des consultations a été calculé en divisant le nombre d'actes ${ }^{8}$ (consultations et visites) par le temps en libéral. Le calcul donne une approximation du nombre de patients vus par le MG en une heure ${ }^{9}$, pour une activité

8 Nombre des consultations et visites en 2013 (données RIAP); a été divisé par le nombre de semaines de travail annuel, estimé à 46,7 en moyenne (Jakoubovitch, 2012). Nous avons aussi enlevé les observations pour lesquelles ce ratio prenait des valeurs inférieures à 0,5 ou supérieure à 6 (ce qui correspond à 43 MG sur 1553 , soit $2,7 \%$ ).

$9 \quad$ Ce rythme intègre des temps que les MG consacrent à leur cabinet sans être nécessairement en présence d'un patient et agrège visites à domiciles et consultations. 
régulière lissée sur la semaine. Nous obtenons un rythme moyen d'environ 2,2 actes par heure.

Compte tenu des caractéristiques démographiques et professionnelles des $\mathrm{MG}^{10}$ ainsi que celles de leur lieu d'installation ${ }^{11}$, les MG des zones les moins dotées ont un rythme de travail significativement plus soutenu que leurs confrères, d'environ $7 \%(0.17 / 2.16)$ - la différence est significative au seuil de $5 \%$ (tableau 3.3).

Tableau 3.3. Rythme de consultation et formation, régressions linéaires multiples

\begin{tabular}{|c|c|c|c|c|}
\hline & \multicolumn{2}{|c|}{$\begin{array}{l}\text { Rythme de consultation } \\
\qquad \mathrm{N}=1463\end{array}$} & \multicolumn{2}{|c|}{$\begin{array}{c}\text { Formation } \\
\mathrm{N}=1378 \\
\end{array}$} \\
\hline & Valeur & p-valeur & Valeur & p-valeur \\
\hline Constante & 2,16 & $<0,01$ & 10,41 & $<0,01$ \\
\hline Zones les moins dotées & $\mathbf{0 , 1 7}$ & $\mathbf{0 , 0 2}$ & $-1,31$ & $\mathbf{0 , 0 3}$ \\
\hline \multicolumn{5}{|l|}{ Age } \\
\hline$<50$ ans & $-0,18$ & $<0,01$ & $-0,61$ & 0,18 \\
\hline$>58$ ans & $-0,06$ & 0,28 & $-0,63$ & 0,15 \\
\hline Femme & $-0,30$ & $<0,01$ & 0,45 & 0,27 \\
\hline Secteur 2 ou non conventionné & $-0,54$ & $<0,01$ & 0,11 & 0,87 \\
\hline Exercice en groupe / MSP & $\mathbf{0 , 2 0}$ & $<0,01$ & 1,16 & $<0,01$ \\
\hline Zone rurale & $-0,08$ & 0,19 & 0,49 & 0,34 \\
\hline Paris et Hauts-de-Seine & 0,14 & 0,19 & $-2,04$ & $\mathbf{0 , 0 2}$ \\
\hline \multicolumn{5}{|l|}{ Niveau de vie de la commune } \\
\hline Q1 & 0,02 & 0,65 & $-0,90$ & 0,04 \\
\hline Q4 & $-0,14$ & $\mathbf{0 , 0 1}$ & 0,26 & 0,58 \\
\hline \multicolumn{5}{|l|}{ Caractéristiques de la patientèle } \\
\hline Parts des patients $<16$ ans & 1,69 & $<0,01$ & $-2,77$ & 0,45 \\
\hline Part de patients $\geq 60$ ans & $-1,02$ & $<0,01$ & $-5,79$ & $\mathbf{0 , 0 2}$ \\
\hline Parts des patients CMU & 1,12 & $<0,01$ & $-3,43$ & 0,22 \\
\hline $\mathrm{R}^{2}$ & \multicolumn{2}{|c|}{$\mathbf{0 , 0 1}$} & \multicolumn{2}{|c|}{$\mathbf{0 , 0 3}$} \\
\hline
\end{tabular}

Champ • Généralistes ayant accepté la communication de leur RIAP, soit 1533 médecins sur 1712 répondants, France métropolitaine.

Source - DREES, URPS-ML et ORS PACA, Pays de la Loire et Poitou-Charentes, panel d'observation des pratiques et des conditions d'exercice en médecine générale, avril-juillet 2014.

Note • Pour la formation, la variable expliquée est le nombre de demi-journées de formation que les médecins déclarent avoir suivis au cours des 12 derniers mois (en 2012), les réponses ont été élaguées (non prise en compte des généralistes déclarant plus de 3 demi-journées de formation, soit 5,4\% des répondants).

Le modèle estimé pour le rythme indique incidemment quelques autres résultats intéressants, notamment le fait que les MG « jeunes » (âgés de moins de 50 ans), les MG femmes, les MG de secteur 2 offrent des consultations de durée plus longue - puisque le rythme est plus faible. On retrouve ainsi des résultats mis en évidence par ailleurs dans la littérature (Jakoubovitch, 2012 ; Jefferson, 2013 ; Hall, 1994 ; Roter, 1991 ; Videau, 2010 ; Wiggers, 1997 ; Buchan, 1973).

10 Âge, sexe, mode d'exercice et de conventionnement.

11 Exercice dans la zone rurale, à Paris ou dans les Hauts-de-Seine, niveau de vie de la commune d'activité, caractéristiques de la patientèle. 
Parmi les sacrifices à consentir pour les MG des zones les moins dotées, le temps consacré à la formation continue est une autre possible « variable d'ajustement » pour un MG contraint par le manque de temps. Effectivement, plus d'un cinquième des MG des zones les moins dotées $(21,0 \%)$ déclarent $n$ 'avoir participé à aucune demi-journée de formation continue au cours des douze derniers mois, alors que cette proportion est nettement plus faible $(13,3 \%)$ parmi leurs confrères des autres zones ( $\mathrm{p}$-valeur=0,04). Toutes choses égales par ailleurs, les MG des zones les moins dotées ont un déficit moyen de 1,31 demi-journée de formation par rapport à leurs collègues des autres zones (tableau 3.3).

\section{Des pratiques différentes dans les zones les moins dotées?}

\subsection{Des différences de prescriptions médicales}

Grâce aux Relevés Individuels d'Activité et de Prescription (RIAP, cf. annexe 3), on a pu calculer pour chaque médecin, et agrégés sur son activité d'une année, des montants de prescription par acte en produits pharmaceutiques, en soins infirmiers, en soins de kinésithérapeutes, en examens biologiques, etc. En utilisant des régressions tenant compte des différences systématiques de patientèle (tableau 4.1), il ressort de la comparaison que les MG des zones les moins dotées prescrivent moins souvent de soins infirmiers et de kinésithérapeutes.

Cet écart pourrait s'expliquer cependant par la sous-densité de ces deux professions paramédicales, la rareté de l'offre de médecins sur un territoire étant souvent associée à une rareté parallèle des professions paramédicales. Lorsque les différences de consommation de soins infirmiers sont étudiées à niveau d'APL infirmiers donné, l'écart n'est plus significatif, ce qui semble en effet confirmer l'hypothèse que l'écart de recours à ces soins s'explique en partie par leur pénurie relative sur le territoire. En revanche, ce même phénomène de disparition de la significativité n'est pas à l'œuvre pour les masseurs-kinésithérapeutes, ce qui suggère qu'il existe de véritables différences de prescriptions que l'on ne pourra pas cette fois imputer à la sous-densité parallèle de l'offre de masso-kinésithérapie. Il est effectivement possible que les médecins perçoivent les soins de kinésithérapie comme plus optionnels, et de ce fait ajustent leurs pratiques de prescriptions au contexte local de l'offre de soins (prescriptions fréquentes en zones bien dotées/ prescriptions plus parcimonieuses en zone moins dotées) ; ce qui serait moins le cas pour les actes infirmiers, dont l'usage est plus normé par des recommandations officielles.

Enfin, les MG de zone moins dotées recourent plus volontiers à certains médicaments : par exemple aux antibiotiques et aux antidiabétiques. On pourrait imaginer que ce type de prescriptions chez les MG des zones moins dotées reflète leur volonté accrue de prévenir et stabiliser des maladies dans un contexte où un second recours en cabinet ou dans un service d'urgences, en cas de complications, promet d'être relativement plus compliqué (Ici, on a deux champs de pathologie de nature assez différente, aiguës pour les antibiotiques, mais aussi chroniques avec un risque de déstabilisation, comme c'est le cas du diabète). 
Tableau 4.1. Remboursements des soins et médicaments prescrits

\begin{tabular}{|c|c|c|c|c|c|c|c|c|}
\hline & \multicolumn{2}{|c|}{$\begin{array}{c}\text { Remboursement soins } \\
\text { infirmiers, par acte } \\
\text { N=1527 }\end{array}$} & \multicolumn{2}{|c|}{$\begin{array}{c}\text { Remboursement soins } \\
\text { masseurs-kinésithérapeutes, } \\
\text { par acte } \\
\mathrm{N}=\mathbf{1 5 2 7}\end{array}$} & \multicolumn{2}{|c|}{$\begin{array}{l}\text { Prescription des } \\
\text { antibiotiques, boites par acte } \\
\mathrm{N}=1527\end{array}$} & \multicolumn{2}{|c|}{$\begin{array}{c}\text { Prescription des } \\
\text { antidiabétiques oraux, } \\
\text { boites par acte } \\
\mathrm{N}=\mathbf{1 5 2 7}\end{array}$} \\
\hline & Valeur & p-valeur & Valeur & p-valeur & Valeur & p-valeur & Valeur & p-valeur \\
\hline Constante & $\mathbf{0 , 4 7}$ & $<0,01$ & 11,54 & $<0,01$ & 0,06 & $<0,01$ & 0,15 & $<0,01$ \\
\hline Zones les moins dotées & $-1,50$ & 0,17 & $-1,12$ & $\mathbf{0 , 0 3}$ & 0,02 & 0,0747 & $\mathbf{0 , 0 4}$ & $<0,01$ \\
\hline $\begin{array}{l}\text { APL infirmiers, Q1 } \\
\text { APL kinésithérapeutes, Q1 }\end{array}$ & $\begin{array}{c}-6,51 \\
- \\
\end{array}$ & $\begin{array}{c}<0,01 \\
- \\
\end{array}$ & $\begin{array}{c}- \\
-3,96 \\
\end{array}$ & $\begin{array}{c}- \\
<0,01 \\
\end{array}$ & $\begin{array}{l}- \\
-\end{array}$ & $\begin{array}{l}- \\
-\end{array}$ & - & $\begin{array}{l}- \\
-\end{array}$ \\
\hline $\begin{array}{l}\text { Age } \\
<50 \text { ans } \\
>58 \text { ans }\end{array}$ & $\begin{array}{l}-1,25 \\
-0,68\end{array}$ & $\begin{array}{l}\mathbf{0 , 0 4} \\
0,39\end{array}$ & $\begin{array}{r}0,06 \\
\mathbf{- 1 , 7 9}\end{array}$ & $\begin{array}{r}0,90 \\
<0,01\end{array}$ & $\begin{array}{l}-0,01 \\
-0,01\end{array}$ & $\begin{array}{l}0,18 \\
0,51\end{array}$ & $\begin{array}{r}-\mathbf{0 , 0 3} \\
0,00\end{array}$ & $\begin{array}{c}<\mathbf{0 , 0 1} \\
0,84\end{array}$ \\
\hline $\begin{array}{l}\text { Femme } \\
\text { Secteur } 2 \text { ou non-conventionné } \\
\text { Exercice en groupe/MSP }\end{array}$ & $\begin{array}{l}-0,55 \\
-4,17 \\
1,11\end{array}$ & $\begin{array}{c}0,41 \\
<\mathbf{0 , 0 1} \\
0,10\end{array}$ & $\begin{array}{c}0,75 \\
-0,32 \\
\mathbf{0 , 9 9}\end{array}$ & $\begin{array}{c}0,10 \\
0,70 \\
<\mathbf{0 , 0 1}\end{array}$ & $\begin{array}{l}0,00 \\
\mathbf{- 0 , 0 7} \\
0,01\end{array}$ & $\begin{array}{c}0,59 \\
<\mathbf{0 , 0 1} \\
0,34\end{array}$ & $\begin{array}{l}\mathbf{- 0 , 0 2} \\
-\mathbf{0 , 0 8} \\
0,01\end{array}$ & $\begin{array}{c}<\mathbf{0 , 0 1} \\
<\mathbf{0 , 0 1} \\
0,17\end{array}$ \\
\hline $\begin{array}{l}\text { Zone rurale } \\
\text { Paris et Hauts-de-Seine }\end{array}$ & $\begin{array}{l}-\mathbf{1 , 8 2} \\
2,70 \\
\end{array}$ & $\begin{array}{l}\mathbf{0 , 0 3} \\
0,14\end{array}$ & $\begin{array}{l}\mathbf{- 1 , 2 9} \\
0,57 \\
\end{array}$ & $\begin{array}{c}<\mathbf{0 , 0 1} \\
0,66 \\
\end{array}$ & $\begin{array}{l}0,03 \\
0,10\end{array}$ & $\begin{array}{l}<0,01 \\
<0,01\end{array}$ & $\begin{array}{l}0,02 \\
\mathbf{0 , 0 4} \\
\end{array}$ & $\begin{array}{l}0,09 \\
\mathbf{0 , 0 2} \\
\end{array}$ \\
\hline $\begin{array}{l}\text { Niveau de vie de la commune } \\
\text { Q1 } \\
\text { Q4 }\end{array}$ & $\begin{array}{l}-0,63 \\
-0,22\end{array}$ & $\begin{array}{l}0,50 \\
0,72\end{array}$ & $\begin{array}{l}-0,79 \\
\mathbf{1 , 5 7}\end{array}$ & $\begin{array}{r}0,10 \\
<0,01\end{array}$ & $\begin{array}{l}-0,01 \\
\mathbf{0 , 0 2}\end{array}$ & $\begin{array}{r}0,55 \\
<0,01\end{array}$ & $\begin{array}{l}\mathbf{0 , 0 3} \\
0,00\end{array}$ & $\begin{array}{c}<\mathbf{0 , 0 1} \\
0,52\end{array}$ \\
\hline $\begin{array}{l}\text { Caractéristiques de la patientèle } \\
\text { Part de patients CMU } \\
\text { Part de < } 16 \text { ans dans la patientèle } \\
\text { Part de patients de } 60 \text { ans ou plus }\end{array}$ & $\begin{array}{r}\mathbf{0 , 2 1} \\
-0,07 \\
\mathbf{0 , 5 5}\end{array}$ & $\begin{array}{c}<\mathbf{0 , 0 1} \\
0,19 \\
<\mathbf{0 , 0 1}\end{array}$ & $\begin{array}{l}-0,05 \\
-\mathbf{0 , 1 4} \\
\mathbf{0 , 1 6}\end{array}$ & $\begin{array}{l}0,07 \\
<\mathbf{0 , 0 1} \\
<\mathbf{0 , 0 1}\end{array}$ & $\begin{array}{l}\mathbf{0 , 0 0} \\
\mathbf{0 , 0 0} \\
\mathbf{0 , 0 0}\end{array}$ & $\begin{array}{l}<0,01 \\
<0,01 \\
\mathbf{0 , 0 2}\end{array}$ & $\begin{array}{l}\mathbf{0 , 0 0} \\
0,00 \\
\mathbf{0 , 0 0}\end{array}$ & $\begin{array}{c}<\mathbf{0 , 0 1} \\
0,14 \\
<\mathbf{0 , 0 1}\end{array}$ \\
\hline$\overline{\mathbf{R}^{2}}$ & & & & & & & & \\
\hline
\end{tabular}

Champ • Généralistes ayant communiqué leur RIAP, n'ayant pas l'activité trop faible ( $<109$ actes) ou trop forte $(>12097$ actes) soit 1454 médecins, France métropolitaine. Source • DREES, URPS-ML et ORS PACA, Pays de la Loire et Poitou-Charentes, panel d'observation des pratiques et des conditions d'exercice en médecine générale. 


\subsection{Des différences de pratiques, vues au travers de la ROSP.}

Dans le même esprit que ce qui précède, nous nous sommes demandé si les MG exerçant au sein des zones les moins dotées présentaient des particularités, cette fois du point de vue de leur succès relatif concernant le dispositif ROSP (cf. annexe 3). Les résultats présentés cidessous sont issus de la population représentative de MG de l'échantillon national, mais, en raison de l'objet d'étude, il a fallu légèrement restreindre l'échantillon aux MG pour lesquels une information complémentaire était disponible : il s'agit des MG ayant accepté l'utilisation de leur RIAP à des fins statistiques et ayant renseigné au moins un indicateur de la ROSP en 2013 (N=1470). De plus, $26 \mathrm{MG}$ ont été exclus suite à une activité trop faible (109 actes) ou trop forte (plus de 12097 actes).

Comme l'objectif de cette étude est de caractériser les différences de pratiques des MG, seuls les indicateurs de prise en charge de patients (suivi des pathologies chroniques et prévention) ont été exploités dans le détail.

A la lecture des analyses descriptives (tableau 4.2), l'hypothèse selon laquelle les MG des zones les moins dotées satisferaient moins bien les objectifs de santé publique sélectionnés dans la ROSP n'est globalement pas validée. Sur les indicateurs composites, les moyennes se distinguent légèrement entre les MG des zones les moins dotées et les autres mais rarement au détriment des MG des zones les moins dotées. On constate même que l'indicateur visant les risques liés à l'iatrogénie du patient âgé serait mieux suivi dans les zones les moins dotées ( $\mathrm{p}$ valeur=0.03). En revanche, les indicateurs de la prévention tels la vaccination ou le dépistage de cancer de sein et du col de l'utérus, seraient parmi les indicateurs les moins bien satisfaits par les MG des zones les moins dotées ( $\mathrm{p}$-valeur=0.04). 
Tableau 4.2. Indicateurs ROSP, statistiques descriptives

\begin{tabular}{|c|c|c|c|c|c|c|c|c|}
\hline & & & $\mathbf{N}$ & Maximum théorique & Moyenne & $\begin{array}{c}\text { Zones les } \\
\text { moins dotées }\end{array}$ & $\begin{array}{c}\text { Autres } \\
\text { zones }\end{array}$ & p-valeur \\
\hline \multirow{5}{*}{\multicolumn{2}{|c|}{ Indicateurs composites }} & Suivi des pathologies chroniques & 1403 & 250 & 139,1 & 145,3 & 138,4 & 0,13 \\
\hline & & Prévention & 1384 & 250 & 103,7 & 109,0 & 103,1 & 0,10 \\
\hline & & Diabète & 1378 & 210 & 105,2 & 109,6 & 104,7 & 0,21 \\
\hline & & Vaccination grippe saisonnière & 1285 & 40 & 4,8 & 3,9 & 4,9 & 0,09 \\
\hline & & Iatrogénie & 1363 & 105 & 64,4 & 68,6 & 63,9 & $\mathbf{0 , 0 3}$ \\
\hline \multirow{9}{*}{$\begin{array}{l}\text { Suivi des } \\
\text { pathologies } \\
\text { chroniques }\end{array}$} & \multirow{8}{*}{$\begin{array}{c}\text { Diabète : \% patients } \\
\text { diabétiques }\end{array}$} & ayant 3 ou 4 dosages d'HbAlc/an & 1237 & 30 & 15,3 & 16,6 & 15,1 & 0,21 \\
\hline & & ayant 1 consultation suivi ophtalmologique/an & 1237 & 35 & 10,5 & 9,4 & 10,7 & 0,25 \\
\hline & & à haut risque cardiovasculaire sous statines & 1102 & 35 & 13,5 & 13,3 & 13,6 & 0,83 \\
\hline & & à haut risque cardiovasculaire sous statines + aspirine faible dose & 910 & 35 & 20,2 & 20,7 & 20,1 & 0,66 \\
\hline & & type 2 dont $\mathrm{HbA} 1 \mathrm{c}<8,5 \%$ & 1277 & 15 & 13,3 & 13,3 & 13,3 & 0,88 \\
\hline & & type 2 dont $\mathrm{HbA} 1 \mathrm{c}<7,5 \%$ & 1270 & 25 & 22,2 & 21,9 & 22,2 & 0,57 \\
\hline & & type 2 dont $\mathrm{LDL}<1,5 \mathrm{~g} / \mathrm{L}$ & 1269 & 10 & 8,2 & 8,2 & 8,2 & 0,97 \\
\hline & & type 2 dont $\mathrm{LDL}<1,3 \mathrm{~g} / \mathrm{L}$ & 1263 & 25 & 20,5 & 20,4 & 20,5 & 0,84 \\
\hline & HTA & $\%$ patients traités par anti-HTA ayant $\mathrm{TA}=140 / 90 \mathrm{~mm} / \mathrm{Hg}$ & 1287 & 40 & 39,7 & 39,5 & 39,7 & 0,42 \\
\hline \multirow{8}{*}{ Prévention } & \multirow{2}{*}{ Vaccination } & $\%$ patients $>65$ ans vaccinés contre grippe saisonnière & 1271 & 20 & 3,8 & 3,1 & 3,9 & 0,14 \\
\hline & & $\%$ patients $16-64$ ans en ALD vaccinés contre grippe saisonnière & 1125 & 20 & 1,2 & 0,9 & 1,3 & 0,11 \\
\hline & \multirow{3}{*}{$\begin{array}{l}\text { Iatrogénie chez les } \\
\text { personnes âgées }\end{array}$} & $\%$ patients $>65$ ans traités par vasodilatateurs & 1286 & 35 & 34,4 & 34,2 & 34,4 & 0,47 \\
\hline & & $\%$ patients $>65$ ans traités par benzodiazépines à demi-vie longue & 1286 & 35 & 17,3 & 20,1 & 17,0 & $<0,01$ \\
\hline & & $\%$ patients $>65$ ans traités par benzodiazépines pendant $>12$ semaines & 1360 & 35 & 15,7 & 15,4 & 15,8 & 0,78 \\
\hline & Cancer du sein & $\%$ patientes (50-74 ans) participant au dépistage cancer du sein & 1322 & 35 & 7,5 & 8,5 & 7,3 & 0,23 \\
\hline & Cancer du col de l'utérus & $\%$ patientes ( $25-65$ ans) ayant bénéficié d' 1 frotti $/ 3$ ans & 1368 & 35 & 7,6 & 5,9 & 7,8 & $\mathbf{0 , 0 4}$ \\
\hline & Antibiothérapie & $\begin{array}{l}\text { Nb prescriptions (chez patients 15-65 ans sans ALD)/an pour } 100 \\
\text { patients }\end{array}$ & 1378 & 35 & 21,7 & 23,1 & 21,5 & 0,23 \\
\hline
\end{tabular}

Champ • Généralistes ayant renseigné au moins un indicateur de la Rémunération sur Objectifs de santé Publique (ROSP), n’ayant pas l'activité trop faible (<109 actes) ou trop forte (>12 097 actes) soit 1454 médecins, France métropolitaine.

Source $•$ DREES, URPS-ML et ORS PACA, Pays de la Loire et Poitou-Charentes, panel d'observation des pratiques et des conditions d'exercice en médecine générale, mars 2014.

Lecture • Le MG réussi mieux la ROSP si le nombre de points atteints est élevé. 
Au-delà des statistiques descriptives (sensibles aux effets de composition), les régressions présentées dans l'Annexe 4 cherchent à identifier les caractéristiques des MG (puis les caractéristiques de la patientèle) qui sont associées à leur réussite aux indicateurs de la ROSP ${ }^{12}$. Par exemple, à autres caractéristiques identiques (Tableau A4.3), les MG femmes obtiennent un nombre de points plus élevé en ce qui concerne le dépistage du cancer du sein et du col utérin. Les MG exerçant en cabinet de groupe ou en MSP ont plus de points ROSP que leurs confrères qui exercent seuls. Les MG ayant une patientèle plus jeune remplissent plus souvent les objectifs de la ROSP. Au contraire, les MG ayant une part plus importante de patients CMU obtiennent moins de points en prévention et en suivi des pathologies chroniques (Tableau A4.2).

Toutes choses égales par ailleurs, le seul indicateur qui montre un lien robuste entre la densité médicale et la réussite à la ROSP est celui de dépistage du cancer de col de l'utérus : les MG installés dans les zones les moins dotées ont 2,86 points de moins que leurs confrères, ce qui pourrait révéler une relative difficulté avec cet objectif chez les MG des zones les moins dotées (mais nous y reviendrons, l'accès aux soins prescrits dans ces zones y est probablement plus difficile). Par ailleurs, les MG des zones les moins dotées réussissent moins souvent l'objectif de consultation annuelle de suivi ophtalmique chez les patients diabétiques. Or, une fois contrôlé par l'offre de soins ophtalmologiques et les caractéristiques de la patientèle, cet effet n'est plus significatif (Tableau A4.2).

\subsection{Les suivis gynécologiques}

Les analyses précédentes ont révélé que l'item relatif à la prévention des cancers du col de l'utérus était significativement «moins réussi » par les MG des zones les moins dotées. Dans cette partie, nous explorons plus avant les pratiques des MG dans le domaine des suivis gynécologiques en exploitant la vague 2 du troisième Panel soit l'échantillon des 1266 MG ayant répondu à cette vague et pour lesquels nous disposons des données issues du RIAP.

Tout comme dans les parties précédentes, les analyses présentées ci-dessous tiennent compte des caractéristiques démographiques et professionnelles des MG ainsi que celles de leur lieu d'installation. La spécificité du sujet traité ici nous a conduit à prendre en considération la densité en gynécologues dans les zones où sont implantés les cabinets des MG enquêtés (nous utilisons pour cela l'indicateur d'APL gynécologue ${ }^{13}$ ) ainsi que l'âge de la patientèle (pour chaque MG, nous disposons de la part de la patientèle âgée de 16 à 59 ans).

\subsubsection{Densité en généralistes et densité en gynécologues}

Plus de la moitié (51,5\%) des MG exerçant dans les zones les moins dotées fait partie des $25 \%$ de MG dont l'APL gynécologue de la commune d'exercice est le plus bas. Si l'on considère, à l'opposé, le groupe constitué des $25 \%$ des MG dont l'APL-gynécologue de la commune d'exercice est le plus haut, il ne contient plus que 6,2\% de MG exerçant dans les zones les moins dotées mais $27,1 \%$ de MG des autres zones. Ainsi, il apparaît que les zones les moins

12 Compte tenu du grand nombre des valeurs manquantes quant aux indicateurs de la ROSP, un modèle tenant compte de biais de sélection a été testé : les résultats y étaient les mêmes que ceux présentés ci-dessous.

13 Cet indicateur est construit selon la même méthodologie que celle de l'APL des MG, présentée dans la partie 2 et en annexe 1. A chaque médecin exerçant en France, correspond la valeur que prend cet indicateur dans la commune où se situe son cabinet. 
dotées en gynécologues ${ }^{14}$ et les zones les moins dotées en MG se recoupent largement. Ceci entre en cohérence avec une approche «populationnelle » qui exprime effectivement un cumul des difficultés : les zones peu dotées en MG sont également peu dotées en gynécologues : $68 \%$ de la population dont l'accessibilité aux MG est « faible » a également une accessibilité aux gynécologues « faible » (DREES, 2016).

Ces recoupements pourraient être à l'origine d'une plus grande difficulté des MG des zones moins denses à valider l'item de la ROSP relatif au dépistage du cancer du col de l'utérus. En effet, pour évaluer cet item, n'entre en compte que la proportion de patientes du médecin traitant ayant bénéficié d'un dépistage sans regard sur son prescripteur. La vague 2 interrogeait les MG sur la fréquence à laquelle ils pratiquent un frottis (geste préalable au dépistage du cancer du col de l'utérus). Il apparaît un net report de ce geste entre généralistes et gynécologues suivant la densité en gynécologues (tableau 4.3). Les MG des zones moins denses, étant beaucoup plus massivement que les autres installés dans des zones de faible densité en gynécologues, ils ne bénéficient donc moins de l'appui des gynécologues pour pouvoir valider l'indicateur : un report vers le MG est visible, mais celui-ci n'est sans doute pas complet.

Tableau 4.3. Fréquence de réalisation d'un frottis cervico-vaginal par un généraliste

\begin{tabular}{|c|c|c|c|c|c|}
\hline \multirow[b]{2}{*}{$\%$ colonnes } & \multicolumn{2}{|c|}{ APL généralistes } & \multicolumn{3}{|c|}{ APL gynécologue } \\
\hline & $\begin{array}{l}\text { Zones les moins } \\
\text { dotées en } \\
\text { généralistes } \\
(\mathbf{N}=127)\end{array}$ & $\begin{array}{l}\text { Autres zones } \\
\qquad(\mathrm{N}=1139)\end{array}$ & $\begin{array}{l}\text { Zones les moins dotées } \\
\text { en gynécologues (Q1) } \\
(\mathbf{N}=315)\end{array}$ & $\begin{array}{c}\text { Zones intermédiaires } \\
(\mathbf{Q 2 - Q 3 )} \\
(\mathrm{N}=629)\end{array}$ & $\begin{array}{l}\text { Zones les plus dotées } \\
\text { en gynécologues (Q4) } \\
\qquad(\mathrm{N}=314)\end{array}$ \\
\hline Au moins une fois par mois & $38,60 \%$ & $35,90 \%$ & $48,50 \%$ & $33,80 \%$ & $28,80 \%$ \\
\hline Moins d'une fois par mois & $61,40 \%$ & $64,10 \%$ & $51,50 \%$ & $66,20 \%$ & $71,20 \%$ \\
\hline p-valeur (test du chi-2) & \multicolumn{2}{|c|}{0,57} & \multicolumn{3}{|c|}{$<0,01$} \\
\hline \multicolumn{6}{|c|}{$\begin{array}{l}\text { Champ • Répondants à la vague } 2 \text { ayant accepté la communication de leur RIAP, soit } 1266 \text { médecins sur } 1414 \text { répondants, } \\
\text { France métropolitaine. } \\
\text { Source } \text { DREES, URPS-ML et ORS PACA, Pays de la Loire et Poitou-Charentes, panel d'observation des pratiques et des } \\
\text { conditions d'exercice en médecine générale, avril-juillet } 2014 \text {. } \\
\text { Note • La question posée était « Au cours de l'année écoulée, à quelle fréquence en moyenne avez-vous réalisé un frottis } \\
\text { cervico-vaginal ? }\end{array}$} \\
\hline
\end{tabular}

4.3.2. Les patientes des zones moins dotées : un suivi gynécologique moins régulier?

La vague 2 interrogeait les MG du Panel sur la fréquence à laquelle ils effectuent une consultation de suivi gynécologique. Outre l'effet de report que nous venons d'observer, une spécificité des MG exerçant dans les zones les moins dotées est de suivre un nombre de patients plus grand que leurs confrères installés dans les autres zones (section 3); pour ces deux raisons,

14 Nous considérons qu'un MG travaille dans une zone parmi les moins dotées en gynécologue s'il fait partie des $25 \%$ de MG dont l'indicateur d'APL-gynécologue est le plus bas. Le seuil a été calculé à partir de la base des 56520 MG exerçant en France en 2010. 
ils devraient donc effectuer un nombre plus élevé de consultations pour suivi gynécologiques ${ }^{15}$. Ce n'est pourtant pas ce qu'on observe (tableau 4.4).

Tableau 4.4. Fréquence de consultation pour les suivis gynécologiques

\begin{tabular}{|c|c|c|c|c|}
\hline & \multicolumn{2}{|c|}{ Suivis de grossesse } & \multicolumn{2}{|c|}{$\begin{array}{c}\text { Suivis gynécologiques } \\
\text { (hors grossesse) }\end{array}$} \\
\hline & $\begin{array}{c}\text { Zones les } \\
\text { moins dotées } \\
(\mathrm{N}=127)\end{array}$ & $\begin{array}{l}\text { Autres zones } \\
\qquad(\mathbf{N}=1139)\end{array}$ & $\begin{array}{c}\text { Zones les } \\
\text { moins dotées } \\
(\mathrm{N}=127)\end{array}$ & $\begin{array}{l}\text { Autres zones } \\
(\mathrm{N}=1139)\end{array}$ \\
\hline Au moins une fois par jour & $0,22 \%$ & $0,54 \%$ & $10,34 \%$ & $7,71 \%$ \\
\hline Au moins une fois par semaine & $11,12 \%$ & $10,33 \%$ & $46,67 \%$ & $48,12 \%$ \\
\hline Au moins une fois par mois & $29,14 \%$ & $27,64 \%$ & $27,14 \%$ & $26,42 \%$ \\
\hline Au moins une fois par trimestre & $17,58 \%$ & $17,55 \%$ & $9,12 \%$ & $7,64 \%$ \\
\hline Moins/jamais & $41,94 \%$ & $43,63 \%$ & $6,73 \%$ & $9,83 \%$ \\
\hline p-valeur (test du chi-2) & \multicolumn{2}{|c|}{0,99} & \multicolumn{2}{|c|}{0,82} \\
\hline
\end{tabular}

Champ 1266 répondants à la vague 2 du panel pour lesquels on dispose des données RIAP

Source • DREES, Unions régionales des professionnels de santé-médecins libéraux (URPS-ML), Observatoires régionaux de la santé (ORS), Panel d'observation des pratiques et des conditions d'exercice en médecine générale, décembre 2014-avril 2015.

Lecture $11,12 \%$ des médecins des zones les moins dotées déclarent avoir vu une patiente pour un suivi de grossesse au moins une fois par semaine au cours de l'année écoulée.

Note - Les questions relatives à la fréquence des suivis de grossesse et des suivis gynécologiques sont : «Au cours de l'année écoulé, à quelle fréquence avez-vous en moyenne vu une patiente pour le suivi régulier d'une grossesse ? » et « Au cours de l'année écoulé, à quelle fréquence avez-vous en moyenne vu une patiente pour un motif gynécologique ?».

Les MG ont également été interrogés sur la fréquence à laquelle ils effectuent certains gestes gynécologiques (examen clinique des seins, toucher vaginal, pose d'un spéculum, consultation pour une pathologie mammaire...). Les résultats sur cet ensemble de pratiques sont en cohérence avec ce que nous venons de remarquer pour les suivis en général. Il n'apparaît pas d'écarts significatifs pour ces fréquences entre zones moins dotées et autres zones, ou bien, lorsque l'écart est significatif, la fréquence est plus faible dans les zones les moins dotées ${ }^{16}$. Ainsi, ces patientes étant plus nombreuses à fréquenter le même $\mathrm{MG}$, et puisque celui-ci ne pratique pas plus souvent de consultations à caractère gynécologique, nous formulons l'hypothèse qu'elles bénéficient d'un suivi gynécologique moins soutenu.

Des questions du Panel permettent aussi d'aborder la question du contenu des consultations pour des suivis gynécologiques sous un angle qualitatif. Il apparaît que les formations des MG des zones moins denses relatives à la gynécologie sont légèrement plus importantes, en moyenne, que celles de leurs confrères des autres zones. Les proportions de MG déclarant avoir un diplôme universitaire en gynécologie obstétrique ou avoir suivi une formation médicale continu dans ce domaine sont comparables (autour de $25 \%$ ) et il en est de même pour ceux ayant effectué un stage hospitalier de second cycle (externat) en gynécologie-obstétrique. déclare que les suivis de grossesse et les suivis gynécologiques hors grossesse font partie de leur mission : 92,4\% des MG de zones moins dotées déclarent que les suivis de grossesse font partie de leurs missions, alors c'est le cas pour $83,2 \%$ de leurs confrères des autres zones ( $p$-valeur $<0,01$ ). La même question concernant les suivis gynécologiques hors grossesse recueille $93,6 \%$ d'acquiescement parmi les médecins des zones moins dotées et $88,3 \%$ pour les autres ( $\mathrm{p}$-valeur $=0,07$ ).

$16 \quad$ C'est le cas par exemple pour l'examen clinique des seins. 
Cependant, une proportion significativement plus élevée, au seuil de $10 \%$, de MG des zones sous-denses déclare avoir effectué un stage hospitalier de gynécologie-obstétrique de 3ème cycle (internat) : ils sont 37,2 \% dans les zones moins dotées, mais seulement 29,5\% dans les autres zones.

Les MG sont aussi interrogés sur les fréquences des échanges qu'ils ont avec d'autres professionnels de santé à propos des suivis gynécologiques. Que ce soit avec d'autres MG, des gynécologues (médical ou obstétriciens), des sages-femmes, des professionnels de PMI ou encore des radiologues (dans le cadre du dépistage du cancer du sein), les MG des zones les moins dotées ne déclarent pas des fréquences d'échanges inférieures. De ce point de vue, on ne peut donc pas dire qu'ils travaillent d'une manière plus isolée que leurs confrères.

Enfin, nous avons vu (section 3.2) que le rythme des consultations est significativement plus soutenu dans les zones les moins dotées. Des questions sur les thèmes abordés pendant les consultations de suivi de grossesse permettent de donner corps à ce résultat : les MG des zones moins dotées sont significativement moins nombreux à aborder systématiquement ou souvent la question de la consommation de tabac ou d'alcool ${ }^{17}$ ou des questions d'ordre psychologique avec la femme enceinte ${ }^{18}$.

63,6\% des MG des zones moins dotées déclarent aborder systématiquement ou souvent la question de la consommation d'alcool avec des patientes enceintes alors qu'ils sont $74,9 \%$ à déclarer le faire dans les autres zones. (la p-valeur $<0,01$ ). En ce qui concerne la consommation de tabac, les pourcentages sont respectivement égaux à $89,5 \%$ et $94,1 \%$ (p-valeur $=0,04)$.

$18 \quad 64,0 \%$ des MG des zones moins dotées déclarent interroger systématiquement ou souvent la patiente sur son état psychologique vis-à-vis de la grossesse alors qu'ils sont $80,1 \%$ à le faire dans les autres zones (pvaleur $<0,01)$. Concernant l'anxiété lié à l'arrivée d'un nouvel enfant, les pourcentages sont respectivement égaux à $59,8 \%$ et $74,03 \%$ (p-valeur $<0,01)$. Enfin, concernant le sentiment d'isolement social, nous trouvons respectivement : $34,9 \%$ et $47,4 \%$ ( $p$-valeur $=0,04)$. 


\section{Conclusion}

Les acteurs de soins du premier recours contribuent de manière importante à la réduction des inégalités socioéconomiques et géographiques (Ferrer, 2005 ; Macinko, 2003 ; Starfield, 2005). En France, les MG de ville ont acquis, depuis la loi de 2004 relative à l'assurance maladie, un rôle pivot «d'aiguilleur » dans le parcours de prise en charge du patient. Ce rôle a été ensuite réaffirmé par la loi « hôpital, patients, santé, territoires » de 2009 et la loi santé 2016, plaçant les soins primaires, et les MG au premier chef, au cœur du système de soins. L'étude de leurs comportements et pratiques dans un contexte de raréfaction de l'offre de soins est donc cruciale pour le suivi du fonctionnement du système de soins tout entier.

Les investigations menées dans le cadre de cette étude portent sur le Panel d'observation des pratiques et reposent sur la recherche systématique d'écarts entre les pratiques des MG exerçant dans les zones les moins dotées et les autres MG du Panel. Les résultats tendent à démontrer que les pratiques des MG sont quelquefois affectées par un environnement d'exercice dans les zones les moins dotées. On peut mettre en avant, notamment, une différence sur la durée des consultations - les MG des zones les moins dotées ont plus d'actes à réaliser et travaillent pourtant à peu près le même nombre d'heures, ce qui est en soi un résultat à relever (même si l'on ne sait pas si ce sont les MG des zones moins dotées qui ont tendance à être peu réactifs en termes de temps de travail, ou si ce sont les MG des zones mieux dotées qui saturent leur contrainte de temps, à des niveaux quasi-identiques à celui de leur confrère en zone sous-dense, éventuellement en répondant aussi à des demandes plus ou moins utiles (on peut évoquer ici le phénomène de «demande induite », Evans, 1974). On note aussi quelques différences statistiquement significatives sur les pratiques médicales : usage de certains médicaments, renvoi vers des soins paramédicaux, suivis gynécologique probablement un peu moins réguliers, etc. Cependant, il semble que ces différences ne se traduisent pas par des différences déterminantes pour la qualité des soins fournie aux patients, du moins en ce qui concerne les indicateurs que nous avons à notre disposition : les indicateurs de performances rattachés à la ROSP. Au total, un apport de ces analyses, en l'état, serait d'amener l'information qu'il n'existe pas de fortes différences de prise en charge des patients dans les zones moins dotées.

Il faut évidemment prendre ces résultats avec précaution. D'abord les indicateurs de la ROSP ne sont qu'un petit bout de la lorgnette avec laquelle examiner la qualité des pratiques; on peut même envisager un effort particulier des MG sur ces indicateurs rémunérés, alors que d'autres actions importantes pour la qualité de soins seraient délaissées (Mannion et Davies, 2008). A cet égard, dès lors qu'on examine des indicateurs complémentaires, autres que la ROSP, comme la fréquence et le contenu des consultations de gynécologie, on observe des différences plus nettes. La population d'observation peut elle-même souffrir de certains biais ; les MG du Panel sont statistiquement représentatifs des MG libéraux, mais dans la « disposition » à répondre à une enquête en panel, dédiée à l'observation des pratiques, peut se cacher une sensibilité particulière des répondants aux questions de qualités des soins et, en général, aux questions de santé publique. Avec le panel, il est possible que l'on parte d'une population plutôt " homogène » dans ses motivations, ce qui, ensuite, rend la mise en évidence d'écarts de pratiques plus compliquée. Enfin, les corrélations statistiques ne sont jamais à coup sûr des causalités. Les régressions sont contrôlées par les caractéristiques médecins et patients, et, grâce à cela, les différences systématiques observables de contextes entre zones les moins dotées et zones mieux dotées sont prises en compte pour éviter tout effet de confusion : on prend en considération notamment : l'âge (du médecin), la structure de la patientèle (âge, prévalence de maladies chroniques), quelques éléments socio-économiques. Mais certaines différences systématiques importantes, touchant par exemple aux motivations professionnelles 
des médecins, restent peut-être inobservées ; ce qui pourrait fragiliser nos analyses, en cas de biais systématique.

Une autre limite tient à la transposition de la situation actuelle, celle que nous observons dans nos données, à la question des futures zones de sous-densité ; on peut craindre que les cas de sous-densité constatés pour le moment soient « en deçà » de ce qui va se passer avec l'accroissement de la raréfaction en MG libéraux que l'on peut anticiper pour les années à venir (cf. supra).

Le dernier point à mentionner serait celui des recherches futures. Pour le moment, nous n'avons pas proposé d'études des facteurs pouvant «neutraliser » la relation entre sous-dotation et adaptations négatives des pratiques médicales - lorsqu'elles existent. On peut imaginer que l'organisation du travail en groupe multidisciplinaire, l'emploi d'assistants, l'usage de télémédecine, etc., sont autant d'éléments qui permettent au médecin d'absorber les excédents d'activité et d'atténuer les effets perturbateurs d'un exercice en zone sous-dense (Gagnon, 2007 ; Mousquès, 2011) - c'est en tout cas explicite dans le choix des pouvoirs publics en faveur d'un soutien au regroupement des médecins en "maison de santé ». Pour le moment, les données du Panel utilisées ici sont insuffisantes pour étudier les différences de comportements des médecins installés en MSP (manque de puissance pour les comparaisons); la prochaine version de ce panel, 4, prévoit un sur-échantillonnage en MSP et permettra probablement ces comparaisons. Cela pourrait être le sujet d'un travail complémentaire à venir, exploitant la mise en place et/ou la généralisation de ces nouveaux types d'organisation du travail médical. 


\section{Bibliographie}

Bachelet M., Anguis M. (2017), « Les médecins d'ici à 2040: une population plus jeune, plus féminisée et plus souvent salariée », Études et Résultats, DREES, n¹011, mai, [en ligne] http://drees.solidarites-sante.gouv.fr/IMG/pdf/er1011.pdf

Buchan I.C., Richardson I.M. (1973), « Time Study of Consultations in General Practice », Edinburgh: Scottish Health Service Studies.

Direction de la recherche, de études, de l'évaluation et des statistiques (2016), " Portrait des professionnels de santé », Panoramas de la DREES - Santé, avril, 160 p., [en ligne] https://drees.solidarites-sante.gouv.fr/etudes-et-statistiques/publications/panoramas-de-ladrees/article/portrait-des-professionnels-de-sante-edition-2016

Dormont B., Huber H. (2012), « Vieillissement de la population et croissance des dépenses de santé », Rapport pour l'Institut Montaigne, Institut Montparnasse, juin, 56 p., [en ligne] https://hal.archivesouvertes.fr/hal-01520109/document

Evans R.G. (1974), « Supplier-Induced Demand: Some Empirical Evidence and Implications », in Perlman M.(dir.), The Economics of Health and Medical Care: Proceedings of a Conference held by the International Economic Association at Tokyo, London: Palgrave Macmillan UK, p. 162-173.

Ferrer R.L., Hambidge S.J., Maly R.C. (2005), « The essential role of generalists in health care systems », Annals of Internal Medecine, vol. 142, n , avril, p.691-699.

Freeman G.K., Horder J.P., Howie J.G. et al. (2002), « Evolving general practice consultation in Britain : issues of length and context », British Medical Journal, vol. 324, $n^{\circ} 7342$, avril, p. 880-882.

Jakoubovitch S., Bournot M.-C., Cercier É. et al. (2012), «Les emplois du temps des médecins généralistes », Etudes et Résultats, DREES, n797, mars, [en ligne] https://drees.solidaritessante.gouv.fr/IMG/pdf/er797-2.pdf

Jefferson L., Bloor K., Birks Y. et al. (2013), « Effect of physicians' gender on communication and consultation length: a systematic review and meta-analysis », Journal of Health Services Research Policy, vol.18, $\mathrm{n}^{\circ} 5$, septembre, p.242-248.

Gagnon M.P., Duplantie J., Fortin J.P. et al. (2007), « Exploring the effects of telehealth on medical human resources supply: a qualitative case study in remote regions », BMC Health Services Research, vol. $7, n^{\circ} 6$.

Hall J.A., Irish J.T., Roter D.L. et al. (1994), « Gender in medical encounters: an analysis of physician and patient communication in a primary care setting », Health Psychology, vol. 13, n 5, septembre, p. 384-392.

Holmboe E.S., Wang Y., Tate J.P. et al. (2006), « The effects of patient volume on the quality of diabetic care for Medicare beneficiaries », Medical Care, vol. 44, n12, décembre, p.1073-1077.

Hutton C., Gunn J. (2007), « Do longer consultations improve the management of psychological problems in general practice? A systematic literature review », BMC Health Services Research, vol. 7 $\mathrm{n}^{\circ} 71$.

Lucas-Gabrielli V., Nestrigue C., en collaboration avec Coldefy M. (2016), « Analyse de 
sensibilité de l'accessibilité potentielle localisée (APL) », Document de travail, IRDES, février, 70 p., [en ligne] http://www.irdes.fr/recherche/documents-de-travail/070-analyse-de-sensibilite-de-1accessibilite-potentielle-localisee-apl.pdf

Le Maréchal M., Collange F., Fressard L. et al. (2015), « Design of a national and regional survey among French general practitioners and method of the first wave of survey dedicated to vaccination », Médecine et Maladies Infectieuses, vol. 45, n¹0, octobre, p. 403-410.

Macinko J., Starfield B., Shi L. (2003), « The contribution of primary care systems to health outcomes within Organization for Economic Cooperation and Development (OECD) countries, 1970-1998 », Health Services Research, vol. 38, ${ }^{\circ} 3$, juin, p. 831-65.

Mannion R., Davies H. T. O. (2008), « Payment for performance in health care », British Medical Journal, vol. 336, $\mathrm{n}^{\circ}$ 7639, février, p. 306-308.

Millien C., Chaput H., Cavillon M. (2018), «La moitié des rendez-vous sont obtenus en 2 jours chez le généraliste, en 52 jours chez l'ophtalmologiste », Études et résultats, DREES, n 1085 , octobre.

Mousquès J. (2011), « Le regroupement des professionnels de santé de premiers recours : quelles perspectives économiques en termes de performance ? », Revue française des affaires sociales, $\mathrm{n}^{\circ} 2-3$, p. 253-275.

OECD Health Statistics (2016), OECD, [en ligne] http://www.oecd.org/els/health-systems/healthdata.htm

Roter D., Lipkin M. Jr, Korsgaard A. (1991), " Sex differences in patients' and physicians' communication during primary care medical visits », Medical Care, vol. 29, n 11 , novembre, p. $1083-$ 1093.

Starfield B., Shi L., Grover A. et al. (2005), « The effects of specialist supply on populations' health : assessing the evidence », Health Affairs. Proj. Hope, Suppl Web Exclusives, W5-97-W5-107, janvierjuin.

Ventelou B., Rolland S., Verger P. (2010), « An Examination of GPs' Rhythm of Practice: A Casevignette to Study the Substitution Effect between the Length of Consultation and Drug Prescribing Practices », Healthcare Policy, vol. 5, n 4, mai, p. 58-68.

Vergier N. (2016), « Accessibilité aux professionnels de santé libéraux : des disparités géographiques variables selon les conditions tarifaires », Études et Résultats, DREES, n 970, juillet, [en ligne] http://drees.social-sante.gouv.fr/IMG/pdf/er_970.pdf

Vergier N., Chaput H. (2017), « Déserts médicaux : comment les définir ? Comment les mesurer ?», Les Dossiers de la DREES, n 17 , mai, [en ligne] http://drees.social-sante.gouv.fr/IMG/pdf/dd17.pdf

Videau Y., Saliba-Serre B., Paraponaris A. et al. (2010), «Why patients of low socioeconomic status with mental health problems have shorter consultations with general practitioners », Journal of Health Services Research and Policy, vol. 15, n² 2, avril, p.76-81.

Wiggers J.H., Sanson-Fisher R. (1997), « Duration of general practice consultations: association with patient occupational and educational status », Social Science and Medicine, vol. 44, n 7, avril, p.925934.

Zyzanski S.J., Stange K.C., Langa D. et al. (1998), « Trade-offs in high-volume primary care practice », The Journal of Family Practice, vol. 46, n5, mai, p.397-402. 


\section{Annexe 1. L'indicateur d'accessibilité potentielle localisée (APL)}

L'indicateur d'accessibilité potentielle localisée (APL) est un indicateur local de densité flottante disponible au niveau de chaque commune, qui tient compte de l'offre et de la demande issues des communes environnantes. Il permet de mieux observer les différences d'accessibilité au niveau local, en mettant en évidence des disparités qu'un indicateur usuel de densité, calculé sur un zonage plus large, aura tendance à masquer, et en minimisant les biais liés au zonage. L'APL classique tient également compte du niveau d'activité des professionnels en exercice, ainsi que de la structure par âge de la population de chaque commune, qui influence les besoins de soins.

Le calcul de l'APL aux MG repose sur l'estimation de plusieurs paramètres :

- On considère que l'accessibilité aux professionnels de santé diminue avec la distance ; les seuils de décroissance de la distance sont estimés à partir des taux de fréquentation des MG observés dans le système national d'informations inter-régimes de l'Assurance maladie (SNIIR-AM). L'indicateur est très sensible à ce paramètre (Lucas-Gabrielli, 2016), qui reflète l'idée de « distance acceptable » à laquelle on accède aux soins.

- L'activité de chaque MG est calculée à partir du nombre d'actes (consultations et visites) réalisés dans l'année. Elle est considérée comme égale au nombre d'actes réalisés si celui-ci est compris entre 3600 et 6000, et ramené à ces bornes dans le cas contraire.

- Enfin, la demande de soins de MG est différenciée par classe d'âge, d'après les consommations par tranche d'âge disponibles dans les bases de l'Assurance maladie.

Chaque commune (et chaque arrondissement dans les grandes villes) de France métropolitaine se voit attribuer une valeur de l'indicateur d'APL qui correspond au nombre moyen de consultations par an et par habitant (pour être plus précis, l'unité est le nombre de consultations et visites accessibles par habitant standardisé et par an). 


\section{Annexe 2. Robustesse des résultats sur la comparaison des temps de travail.}

\section{A2.1. Aménagement de l'indicateur d'APL}

Appelons indicateur d'APL-effectif une variante de l'indicateur d'APL pour laquelle le calcul de l'offre en consultations intègre chaque $\mathrm{MG}$ à hauteur d'un équivalent temps plein. Nous pouvons définir les médecins exerçant dans les zones les moins dotées comme étant ceux dont l'APL-effectif de la commune d'exercice fait partie des $10 \%$ les plus bas. Cet aménagement de l'indicateur de densité en MG induit un changement du groupe des médecins du panel considérés comme exerçant en zones les moins dotées par rapport à celui obtenu avec l'APL classique.

Tableau A2.1. Nombre d'actes et temps de travail déclarés en fonction de la densité médicale obtenue avec l'APL-effectif

\begin{tabular}{|c|c|c|c|}
\hline & $\begin{array}{c}\text { Nombre d'actes } \\
\text { (1) }\end{array}$ & $\begin{array}{c}\text { Temps de travail (total) } \\
\text { (2) }\end{array}$ & $\begin{array}{l}\text { Temps de travail (activité libérale) } \\
\text { (3) }\end{array}$ \\
\hline $\begin{array}{l}\text { Zones les moins dotées } \\
\text { (construites à partir de l'APL-effectif) }\end{array}$ & 5474 & 56,1 & 48,5 \\
\hline Autres zones & 4894 & 54,7 & 47,8 \\
\hline $\begin{array}{l}\text { Variation en pourcentage (zones sous dotées / } \\
\text { autres zones) }\end{array}$ & $+12,0 \%$ & $+3 \%$ & $+1,0 \%$ \\
\hline p-value (test de Student) & $<0,01$ & 0,22 & 0,45 \\
\hline
\end{tabular}

Champ / Source / Note $\bullet$ cf. tableau 3.1

Lecture • Les temps de travail déclarés des généralistes selon qu'ils exercent ou non dans une des zones les moins dotées ne sont pas significativement différents.

\section{A2.2. Sensibilité des résultats à un changement du seuil permettant de définir les médecins exerçant en zone moins dotées}

De nouveau, la densité en MG est obtenue à l'aide de l'indicateur d'APL ${ }^{19}$. Nous observons qu'un changement du seuil permettant de définir les médecins exerçant dans les zones moins dotées n'affecte pas la non-significativité des écarts de temps de travail déclaré. 
Tableau A2.2. Significativité des différences du temps de travail à l'aune de différents seuils.

\begin{tabular}{|c|c|c|c|c|c|c|c|c|c|c|c|c|}
\hline & \multicolumn{3}{|c|}{$\begin{array}{c}\text { Seuil : } 1 \% \\
\text { Effectif zones les moins dotées : } 19\end{array}$} & \multicolumn{3}{|c|}{$\begin{array}{l}\text { Seuil : } 5 \% \\
\text { Effectif zones les moins dotées : } \mathbf{8 5}\end{array}$} & \multicolumn{3}{|c|}{$\begin{array}{l}\text { Seuil : } 20 \% \\
\text { Effectif zones les moins dotées : } \mathbf{3 2 8}\end{array}$} & \multicolumn{3}{|c|}{$\begin{array}{c}\text { Seuil : } 25 \% \\
\text { Effectif zones les moins dotées : } 419\end{array}$} \\
\hline & $\begin{array}{c}\text { Nombre } \\
\text { d'actes }\end{array}$ & $\begin{array}{c}\text { Temps de } \\
\text { travail (total) }\end{array}$ & $\begin{array}{l}\text { Temps de travail } \\
\text { (activité libérale) }\end{array}$ & \begin{tabular}{|l|l|}
$\begin{array}{l}\text { Nombre } \\
\text { d'actes }\end{array}$ \\
\end{tabular} & $\begin{array}{c}\text { Temps de } \\
\text { travail (total) }\end{array}$ & $\begin{array}{l}\text { Temps de travail } \\
\text { (activité libérale) }\end{array}$ & \begin{tabular}{|l|}
$\begin{array}{l}\text { Nombre } \\
\text { d'actes }\end{array}$ \\
\end{tabular} & $\begin{array}{c}\text { Temps de } \\
\text { travail (total) }\end{array}$ & $\begin{array}{l}\text { Temps de travail } \\
\text { (activité libérale) }\end{array}$ & \begin{tabular}{|l|l|}
$\begin{array}{l}\text { Nombre } \\
\text { d'actes }\end{array}$ \\
\end{tabular} & $\begin{array}{c}\text { Temps de } \\
\text { travail (total) }\end{array}$ & $\begin{array}{l}\text { Temps de travail } \\
\text { (activité libérale) }\end{array}$ \\
\hline Zones les moins dotées & 4854 & 55,8 & 49,2 & 4929 & 56,5 & 48,1 & 5329 & 55,7 & 48,2 & 5320 & 55,5 & 47,9 \\
\hline Autres zones & 4949 & 54,8 & 47,8 & 4949 & 54,7 & 47,8 & 4853 & 54,6 & 47,8 & 4821 & 54,6 & 47,8 \\
\hline p-valeur (test de Student) & 0,39 & 0,70 & 0,60 & 0,90 & 0,24 & 0,80 & $<0,01$ & 0,17 & 0,54 & $<0,01$ & 0,20 & 0,85 \\
\hline \multicolumn{13}{|c|}{ Résultats des régressions linéaires multiples } \\
\hline Zones les moins dotées & $-60,00$ & 0,25 & 0,20 & $-105,00$ & 0,55 & $-0,92$ & 561,00 & 0,91 & 0,17 & 561,00 & 0,70 & $-0,14$ \\
\hline p-valeur (régression) & 0,90 & 0,90 & 0,90 & 0,70 & 0,70 & 0,51 & $<0,01$ & 0,28 & 0,83 & $<0,01$ & 0,36 & 0,85 \\
\hline
\end{tabular}




\section{A2.3. Comparaison d'estimations de temps de consultation.}

Cette partie permet de se focaliser sur les plages horaires du médecin qui sont vraiment dédiées à la rencontre médecin-patient dans le cadre d'une consultation en libéral. Sont d'abord considérés les horaires correspondant au temps de travail dédié à l'activité « médicale » : horaires déclarés pour des activités libérales auxquels on décompte le temps consacré aux tâches administratives (également déclarés par les MG). Ensuite, le temps passé en consultation a été défini comme un solde : il s'agit du temps de travail libéral dont ont été déduits les temps consacrés aux tâches administratives, à la mise à jour des connaissances médicales, à l'activité d'encadrement de jeunes médecins (maître de stage).

Les résultats confirment ceux de la section 3.1 : les MG des zones les moins dotées n'ont pas le temps médical ni celui de consultation plus élevé.

Tableau A2.3. Temps de consultation déclaré en fonction de la densité médicale

\begin{tabular}{lcc|cc}
\hline & \multicolumn{2}{c|}{ Temps médical } & \multicolumn{2}{c}{ Temps de consultation } \\
& \multicolumn{2}{c}{$\mathbf{N = 1 4 9 2}$} & \multicolumn{2}{c}{ N=1481 } \\
\cline { 2 - 6 } & Valeur & p-valeur & Valeur & p-valeur \\
\hline Constante & $\mathbf{4 6 , 6 1}$ & $\mathbf{< 0 , 0 1}$ & $\mathbf{4 2 , 6 4}$ & $<\mathbf{0 , 0 1}$ \\
\hline Zones les moins dotées & $-0,80$ & 0,51 & $-0,48$ & 0,68 \\
\hline Age & & & & \\
$<50$ ans & $\mathbf{- 2 , 1 4}$ & $\mathbf{< 0 , 0 1}$ & $\mathbf{- 1 , 6 2}$ & $\mathbf{0 , 0 2}$ \\
$>$ 58 ans & $\mathbf{- 1 , 9 6}$ & $<\mathbf{0 , 0 1}$ & $\mathbf{- 1 , 5 3}$ & $\mathbf{0 , 0 4}$ \\
\hline Femme & $\mathbf{- 6 , 3 9}$ & $<\mathbf{0 , 0 1}$ & $\mathbf{- 5 , 8 8}$ & $<\mathbf{0 , 0 1}$ \\
Secteur 2 ou non-conventionné & $-0,64$ & 0,60 & $-1,83$ & 0,15 \\
Exercice en groupe/MSP & $-0,08$ & 0,90 & $-0,58$ & 0,37 \\
\hline Zone rurale & 1,26 & 0,18 & 1,13 & 0,25 \\
Paris et Hauts-de-Seine & 0,28 & 0,88 & 0,39 & 0,84 \\
\hline Niveau de vie de la commune & & & & \\
Q1 & 0,77 & 0,34 & 0,66 & 0,43 \\
Q4 & $-0,95$ & 0,21 & $-1,13$ & 0,14 \\
\hline Caractéristiques de la patientèle & & & & \\
Part de patients CMU & $-0,07$ & 0,25 & $-0,07$ & 0,21 \\
Part de < 16 ans dans la patientèle & 0,02 & 0,72 & 0,04 & 0,52 \\
Part de patients de 60 ans ou plus & $-0,01$ & 0,93 & 0,04 & 0,48 \\
\hline $\mathbf{R}^{2}$ & & $\mathbf{0 , 0 8}$ & & $\mathbf{0 , 0 7}$ \\
\hline
\end{tabular}




\section{Annexe 3. Eléments de cadrage : le RIAP et la ROSP}

\section{A3.1. Le Relevé individuel d'activité et de prescriptions (RIAP)}

Le RIAP est un relevé informatique effectué par le système d'Assurance maladie qui relie certaines consommations de soins des assurés sociaux à l'identité du " prescripteur » de ces soins, ici, en l'occurrence, leur MG. Celui-ci contient l'information sur l'activité du MG (consultations, visites à domicile), les caractéristiques de la patientèle (part de patients pour qui le médecin est le médecin traitant) et les prescriptions (pharmacie, soins de kinésithérapie, soins infirmiers...).

\section{A3.2. La Rémunération sur objectifs de santé publique (ROSP)}

La ROSP est un dispositif de paiement à la performance mis en place par la convention nationale liant l'assurance maladie et les médecins libéraux. Depuis 2012, la ROSP concerne tous les MG libéraux conventionnés, sauf ceux qui refusent explicitement cette rémunération supplémentaire. Dans la version 2013, utilisée dans nos analyses, il s'agissait d'un système de 29 indicateurs, organisés autour de deux grandes dimensions : organisation (informatisation) du cabinet médical et qualité de pratique médicale. Dans cette dernière, on distingue trois axes : suivi des pathologies chroniques (notamment diabète, 9 indicateurs), prévention ( 8 indicateurs) et optimisation des prescriptions médicales (principalement la prescription des médicaments génériques, 7 indicateurs).

Les MG perçoivent une rémunération de la part de l'Assurance maladie correspondant soit à la réalisation d'un objectif soit à la progression observée vers cet objectif. Chaque indicateur est indépendant ; les indicateurs et les objectifs ont été fixés avec les représentants des médecins lors des négociations conventionnelles et tiennent compte des recommandations de la Haute Autorité de Santé ainsi que des recommandations internationales. 


\section{Annexe 4. Indicateurs ROSP, régressions linéaires multiples}

Tableau A4.1 Indicateurs composites, régressions multiples

\begin{tabular}{lcc|cc}
\hline & \multicolumn{2}{c|}{$\begin{array}{c}\text { Suivi des maladies } \\
\text { chroniques }\end{array}$} & \multicolumn{2}{c}{ Prévention } \\
& \multicolumn{2}{c}{ N=1403 } & \multicolumn{2}{c}{ N=1384 } \\
\cline { 2 - 6 } & Valeur & p-valeur & Valeur & p-valeur \\
\hline Constante & $\mathbf{1 2 9 , 0 4}$ & $<\mathbf{0 , 0 1}$ & $\mathbf{1 0 0 , 2 3}$ & $<\mathbf{0 , 0 1}$ \\
\hline Zones les moins dotées & 2,79 & 0,57 & 3,54 & 0,32 \\
\hline Age & & & & \\
$<50$ ans & $\mathbf{- 1 1 , 9 0}$ & $\mathbf{< 0 , 0 1}$ & $\mathbf{- 7 , 3 7}$ & $<\mathbf{0 , 0 1}$ \\
$>58$ ans & $\mathbf{- 9 , 2 1}$ & $<\mathbf{0 , 0 1}$ & $\mathbf{- 1 0 , 2 0}$ & $<\mathbf{0 , 0 1}$ \\
\hline Femme & $\mathbf{- 9 , 9 0}$ & $<\mathbf{0 , 0 1}$ & 0,37 & 0,88 \\
Secteur 2 ou non-conventionné & $\mathbf{- 1 4 , 4 0}$ & $\mathbf{0 , 0 4}$ & 6,71 & 0,14 \\
Exercice en groupe/MSP & $\mathbf{9 , 0 4}$ & $\mathbf{< 0 , 0 1}$ & $\mathbf{4 , 2 8}$ & $\mathbf{0 , 0 4}$ \\
\hline Zone rurale & $-1,15$ & 0,78 & $\mathbf{- 1 0 , 2 0}$ & $<\mathbf{0 , 0 1}$ \\
Paris et Hauts-de-Seine & $-10,60$ & 0,22 & $-1,84$ & 0,73 \\
\hline Niveau de vie de la commune & & & & \\
Q1 & $-2,75$ & 0,47 & 0,16 & 0,96 \\
Q4 & $-3,87$ & 0,30 & $\mathbf{5 , 8 6}$ & $\mathbf{0 , 0 2 8}$ \\
\hline Caractéristiques de la patientèle & & & & \\
Part de patients CMU & $\mathbf{0 , 9 7}$ & $<\mathbf{0 , 0 1}$ & $\mathbf{- 1 , 4 4}$ & $<\mathbf{0 , 0 1}$ \\
Part de < 16 ans dans la patientèle & $\mathbf{0 , 8 3}$ & $\mathbf{0 , 0 1}$ & $\mathbf{0 , 8 9}$ & $<\mathbf{0 , 0 1}$ \\
Part de patients de 60 ans ou plus & 0,32 & 0,14 & $-0,05$ & 0,76 \\
\hline $\mathbf{R}^{\mathbf{2}}$ & & $\mathbf{0 , 0 6}$ & & $\mathbf{0 , 1 3}$ \\
\hline
\end{tabular}

Champ • Généralistes ayant renseigné au moins un indicateur de la ROSP, n'ayant pas l'activité trop faible ou trop forte soit 1444 médecins, France métropolitaine.

Source - DREES, URPS-ML et ORS PACA, Pays de la Loire et Poitou-Charentes, panel d'observation des pratiques et des conditions d'exercice en médecine générale. 
Tableau A4.2.1. Indicateurs, suivi des maladies chroniques, régressions multiples

\begin{tabular}{|c|c|c|c|c|}
\hline & \multicolumn{2}{|c|}{$\begin{array}{l}\text { Diabète } \\
\mathrm{N}=1378 \\
\end{array}$} & \multicolumn{2}{|c|}{$\begin{array}{c}\text { \% patients traités par } \\
\text { anti-HTA ayant TA = } \\
140 / 90 \mathrm{~mm} / \mathrm{Hg} \\
\mathrm{N}=1287\end{array}$} \\
\hline & Valeur & p-valeur & Valeur & p-valeur \\
\hline Constante & 93,06 & $<0,01$ & 39,42 & $<0,01$ \\
\hline Zones les moins dotées & 1,64 & 0,70 & $-0,27$ & 0,25 \\
\hline \multicolumn{5}{|l|}{ Age } \\
\hline$<50$ ans & $-11,30$ & $<0,01$ & $-0,24$ & 0,16 \\
\hline$>58$ ans & $-6,54$ & $\mathbf{0 , 0 3}$ & $-0,19$ & 0,27 \\
\hline Femme & $-8,01$ & $<0,01$ & 0,18 & 0,08 \\
\hline Secteur 2 ou non-conventionné & $-3,97$ & 0,47 & $-0,54$ & 0,36 \\
\hline Exercice en groupe/MSP & 7,04 & $<0,01$ & 0,10 & 0,48 \\
\hline Zone rurale & $-3,14$ & 0,38 & 0,24 & 0,08 \\
\hline Paris et Hauts-de-Seine & $-11,00$ & 0,10 & 0,46 & 0,19 \\
\hline \multicolumn{5}{|l|}{ Niveau de vie de la commune } \\
\hline Q1 & $-1,63$ & 0,61 & 0,27 & 0,09 \\
\hline Q4 & $-4,81$ & 0,14 & 0,17 & 0,37 \\
\hline \multicolumn{5}{|l|}{ Caractéristiques de la patientèle } \\
\hline Part de patients CMU & $-0,95$ & $<0,01$ & $-0,01$ & 0,37 \\
\hline Part de $<16$ ans dans la patientèle & $\mathbf{0 , 9 3}$ & $<0,01$ & 0,02 & 0,27 \\
\hline Part de patients de 60 ans ou plus & 0,28 & 0,12 & 0,00 & 0,72 \\
\hline $\mathbf{R}^{2}$ & \multicolumn{2}{|c|}{0,06} & \multicolumn{2}{|c|}{0,01} \\
\hline
\end{tabular}

Champ • Généralistes ayant renseigné au moins un indicateur de la ROSP, n'ayant pas l'activité trop faible ou trop forte soit 1444 médecins, France métropolitaine.

Source - DREES, URPS-ML et ORS PACA, Pays de la Loire et Poitou-Charentes, panel d'observation des pratiques et des conditions d'exercice en médecine générale. 
Tableau A4.2.2. Indicateurs, suivi des maladies chroniques, régressions multiples (suite)

\begin{tabular}{|c|c|c|c|c|c|c|c|c|}
\hline & \multicolumn{8}{|c|}{ \% patients diabétiques } \\
\hline & \multirow{2}{*}{\multicolumn{2}{|c|}{$\begin{array}{c}\begin{array}{c}\text { ayant } 3 \text { ou } 4 \text { dosages } \\
\text { d'HbA1c/an }\end{array} \\
\mathrm{N}=1237 \\
\end{array}$}} & \multirow{2}{*}{\multicolumn{2}{|c|}{$\begin{array}{c}\text { à haut risque cardiovasculaire } \\
\text { sous statines }\end{array}$}} & \multirow{2}{*}{\multicolumn{2}{|c|}{$\begin{array}{l}\text { à haut risque cardiovasculaire sous statines + } \\
\text { aspirine faible dose } \\
\mathrm{N}=910\end{array}$}} & \multirow{2}{*}{\multicolumn{2}{|c|}{$\begin{array}{c}\begin{array}{c}\text { ayant } 1 \text { consultation suivi } \\
\text { ophtalmologique/an }\end{array} \\
\mathbf{N}=1237\end{array}$}} \\
\hline & & & & & & & & \\
\hline & Valeur & p-valeur & Valeur & p-valeur & Valeur & p-valeur & Valeur & p-valeur \\
\hline Constante & 8,51 & $<0,01$ & 8,10 & 0,04 & 16,62 & $<0,01$ & 6,14 & 0,07 \\
\hline Zones les moins dotées & 0,32 & 0,80 & $-1,04$ & 0,45 & 0,73 & 0,63 & $-1,89$ & 0,09 \\
\hline APL ophtalmologues, Q1 & - & - & - & - & - & - & $-2,00$ & $\mathbf{0 , 0 4}$ \\
\hline \multicolumn{9}{|l|}{ Age } \\
\hline$<50$ ans & 1,73 & 0,05 & 0,31 & 0,77 & 2,96 & $\mathbf{0 , 0 2}$ & 0,92 & 0,32 \\
\hline$>58$ ans & $-2,36$ & $<0,01$ & 0,16 & 0,87 & $-1,03$ & 0,37 & $-0,88$ & 0,29 \\
\hline Femme & 0,92 & 0,26 & 0,84 & 0,43 & $-1,71$ & 0,14 & 2,89 & $<0,01$ \\
\hline Secteur 2 ou non-conventionné & 0,43 & 0,80 & 0,61 & 0,78 & 1,22 & 0,60 & 4,64 & 0,02 \\
\hline Exercice en groupe/MSP & 0,90 & 0,21 & 0,34 & 0,70 & 1,40 & 0,15 & $-0,12$ & 0,86 \\
\hline Zone rurale & $-1,40$ & 0,18 & 0,12 & 0,92 & $-0,47$ & 0,73 & 0,44 & 0,68 \\
\hline Paris et Hauts-de-Seine & $-8,61$ & $<0,01$ & 5,10 & $\mathbf{0 , 0 5}$ & $-2,97$ & 0,28 & $-5,04$ & $\mathbf{0 , 0 2}$ \\
\hline \multicolumn{9}{|l|}{ Niveau de vie de la commune } \\
\hline Q1 & $-1,55$ & 0,10 & 0,75 & 0,51 & 0,17 & 0,89 & 0,70 & 0,42 \\
\hline Q4 & $-1,92$ & 0,04 & 1,48 & 0,21 & 0,01 & 0,99 & 1,25 & 0,21 \\
\hline \multicolumn{9}{|l|}{ Caractéristiques de la patientèle } \\
\hline Part de patients CMU & $-0,31$ & $<0,01$ & $-0,16$ & $\mathbf{0 , 0 2}$ & $-0,06$ & 0,54 & $-0,22$ & $<0,01$ \\
\hline Part de $<16$ ans dans la patientèle & 0,32 & $<0,01$ & $\mathbf{0 , 3 6}$ & $<0,01$ & 0,15 & 0,29 & $\mathbf{0 , 2 0}$ & 0,04 \\
\hline Part de patients de 60 ans ou plus & 0,15 & 0,01 & $-0,07$ & 0,33 & 0,01 & 0,94 & 0,06 & 0,36 \\
\hline
\end{tabular}

0,11

0,04

0,03

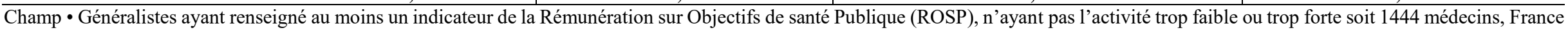
métropolitaine.

Source • DREES, URPS-ML et ORS PACA, Pays de la Loire et Poitou-Charentes, panel d'observation des pratiques et des conditions d'exercice en médecine générale. 
Tableau A4.2.3. Indicateurs, suivi des maladies chroniques, régressions multiples (suite)

$\%$ patients diabétiques de type 2

\begin{tabular}{|c|c|c|c|c|c|c|c|c|}
\hline & \multicolumn{8}{|c|}{ \% patients diabétiques de type 2} \\
\hline & \multicolumn{2}{|c|}{$\begin{array}{c}\text { dont HbA1c }<8,5 \% \\
\text { N=1277 }\end{array}$} & \multicolumn{2}{|c|}{$\begin{array}{c}\text { dont } \mathrm{HbA1c}<7,5 \% \\
\mathrm{~N}=1270\end{array}$} & \multicolumn{2}{|c|}{$\begin{array}{c}\text { dont } \mathrm{LDL}<1,5 \mathrm{~g} / \mathrm{L} \\
\mathrm{N}=1269\end{array}$} & \multicolumn{2}{|c|}{$\begin{array}{c}\text { dont } \mathrm{LDL}<1,3 \mathrm{~g} / \mathrm{L} \\
\mathrm{N}=1263\end{array}$} \\
\hline & Valeur & p-valeur & Valeur & p-valeur & Valeur & p-valeur & Valeur & p-valeur \\
\hline Constante & 13,97 & $<0,01$ & 22,50 & $<0,01$ & 9,21 & $<0,01$ & 22,10 & $<0,01$ \\
\hline Zones les moins dotées & $-0,06$ & 0,83 & $-0,40$ & 0,39 & 0,07 & 0,79 & $-0,04$ & 0,96 \\
\hline \multicolumn{9}{|l|}{ Age } \\
\hline$<50$ ans & $-0,34$ & 0,12 & $-0,21$ & 0,54 & $-0,18$ & 0,33 & $-0,23$ & 0,62 \\
\hline$>58$ ans & 0,14 & 0,53 & 0,15 & 0,69 & $-0,10$ & 0,61 & $-0,25$ & 0,59 \\
\hline Femme & 0,16 & 0,41 & 0,05 & 0,88 & 0,18 & 0,28 & 0,52 & 0,22 \\
\hline Secteur 2 ou non-conventionné & 0,01 & 0,98 & 0,37 & 0,54 & 0,10 & 0,79 & $-0,13$ & 0,90 \\
\hline Exercice en groupe/MSP & 0,33 & 0,08 & 0,18 & 0,54 & 0,08 & 0,61 & 0,27 & 0,49 \\
\hline Zone rurale & 0,17 & 0,50 & $-0,15$ & 0,69 & 0,02 & 0,93 & 0,02 & 0,96 \\
\hline Paris et Hauts-de-Seine & $-0,58$ & 0,41 & $-0,54$ & 0,54 & $-0,66$ & 0,28 & $-0,43$ & 0,74 \\
\hline \multicolumn{9}{|l|}{ Niveau de vie de la commune } \\
\hline Q1 & 0,10 & 0,69 & $-0,21$ & 0,58 & $-0,21$ & 0,29 & $-0,58$ & 0,25 \\
\hline Q4 & $-0,06$ & 0,79 & 0,60 & 0,08 & $-0,37$ & 0,05 & $-0,68$ & 0,16 \\
\hline \multicolumn{9}{|l|}{ Caractéristiques de la patientèle } \\
\hline Part de patients CMU & $-0,03$ & $\mathbf{0 , 0 4}$ & $-0,05$ & 0,08 & 0,00 & 0,88 & $-0,02$ & 0,57 \\
\hline Part de $<16$ ans dans la patientèle & $-0,01$ & 0,57 & 0,00 & 0,91 & $-0,03$ & 0,13 & $-0,04$ & 0,41 \\
\hline Part de patients de 60 ans ou plus & $-0,01$ & 0,32 & 0,00 & 0,88 & $-0,01$ & 0,24 & $-0,02$ & 0,51 \\
\hline $\mathbf{R}^{2}$ & \multicolumn{2}{|c|}{$\mathbf{0 , 0 1}$} & \multicolumn{2}{|c|}{$\mathbf{0 , 0 1}$} & \multicolumn{2}{|c|}{$\mathbf{0 , 0 1}$} & \multicolumn{2}{|c|}{$\mathbf{0 , 0 1}$} \\
\hline
\end{tabular}

Champ • Généralistes ayant renseigné au moins un indicateur de la ROSP, n'ayant pas l'activité trop faible ou trop forte soit 1444 médecins, France métropolitaine. Source • DREES, URPS-ML et ORS PACA, Pays de la Loire et Poitou-Charentes, panel d'observation des pratiques et des conditions d'exercice en médecine générale. 
Tableau A4.3.1 Indicateurs, prévention, régressions multiples

\begin{tabular}{|c|c|c|c|c|c|c|c|c|c|c|}
\hline & \multicolumn{2}{|c|}{$\begin{array}{c}\text { Vaccination grippe } \\
\text { saisonnière } \\
\mathbf{N}=1285 \\
\end{array}$} & \multicolumn{2}{|c|}{$\begin{array}{l}\text { Iatrogénie } \\
\mathrm{N}=1363 \\
\end{array}$} & \multicolumn{2}{|c|}{$\begin{array}{l}\text { \% patientes }(50-74 \text { ans }) \\
\text { dépistage du cancer du } \\
\text { sein } \\
\mathrm{N}=1322\end{array}$} & \multicolumn{2}{|c|}{$\begin{array}{c}\% \text { patientes }(25-65 \text { ans }) \\
1 \text { frotti/3 ans } \\
\text { N=1368 }\end{array}$} & \multicolumn{2}{|c|}{$\begin{array}{c}\text { Prescriptions d'antibiotiques } \\
\text { (15-65 ans sans ALD)/an } \\
\text { pour } 100 \text { patients } \\
\mathrm{N}=1378\end{array}$} \\
\hline & Valeur & p-valeur & Valeur & p-valeur & Valeur & p-valeur & Valeur & p-valeur & Valeur & p-valeur \\
\hline Constante & 4,63 & $<0,01$ & 60,61 & $<0,01$ & 6,07 & $<0,01$ & $\mathbf{9 , 7 8}$ & $<0,01$ & 19,50 & $<0,01$ \\
\hline Zones les moins dotées & $-1,15$ & $\mathbf{0 , 0 3}$ & 2,91 & 0,23 & 0,89 & 0,35 & $-2,78$ & $<0,01$ & 2,14 & 0,16 \\
\hline \multicolumn{11}{|l|}{ Age } \\
\hline$<50$ ans & 0,10 & 0,83 & $-7,30$ & $<0,01$ & 0,81 & 0,28 & 2,54 & $<0,01$ & 0,51 & 0,61 \\
\hline$>58$ ans & $-0,68$ & 0,13 & $-5,65$ & $<0,01$ & $-0,86$ & 0,19 & $-1,11$ & $\mathbf{0 , 0 5}$ & $-0,67$ & 0,51 \\
\hline Femme & $-0,75$ & 0,06 & $-3,07$ & 0,06 & 2,12 & $<0,01$ & 1,98 & $<0,01$ & 2,83 & $<0,01$ \\
\hline Secteur 2 ou non-conventionné & $-2,18$ & $<0,01$ & 3,57 & 0,21 & 0,44 & 0,72 & 6,22 & $<0,01$ & 3,04 & 0,08 \\
\hline Exercice en groupe/MSP & 0,48 & 0,21 & 0,44 & 0,74 & 0,56 & 0,32 & $-0,49$ & 0,35 & 3,46 & $<0,01$ \\
\hline Zone rurale & $-0,29$ & 0,60 & $-1,85$ & 0,32 & $-3,70$ & $<0,01$ & $-1,50$ & $\mathbf{0 , 0 3}$ & $-2,20$ & 0,08 \\
\hline Paris et Hauts-de-Seine & $-2,56$ & $<0,01$ & 0,39 & 0,92 & $-5,56$ & $<0,01$ & $-2,57$ & 0,11 & 6,62 & $<0,01$ \\
\hline \multicolumn{11}{|l|}{ Niveau de vie de la commune } \\
\hline Q1 & $-0,06$ & 0,91 & 0,95 & 0,60 & $-2,14$ & $<0,01$ & $-0,76$ & 0,18 & 2,00 & 0,09 \\
\hline Q4 & $-0,30$ & 0,53 & 4,10 & $\mathbf{0 , 0 2}$ & 0,45 & 0,56 & 2,95 & $<0,01$ & $-0,36$ & 0,73 \\
\hline \multicolumn{11}{|l|}{ Caractéristiques de la patientèle } \\
\hline Part de patients CMU & $-0,03$ & 0,22 & $-0,44$ & $<0,01$ & $-0,26$ & $<0,01$ & $-0,42$ & $<0,01$ & $-0,32$ & $<0,01$ \\
\hline Part de $<16$ ans dans la patientèle & 0,02 & 0,69 & 0,49 & $<0,01$ & $\mathbf{0 , 1 3}$ & 0,04 & 0,15 & $\mathbf{0 , 0 1}$ & 0,10 & 0,27 \\
\hline Part de patients de 60 ans ou plus & 0,03 & 0,32 & 0,00 & 0,96 & 0,04 & 0,27 & $-0,13$ & $<0,01$ & $-0,03$ & 0,66 \\
\hline $\mathbf{R}^{2}$ & \multicolumn{2}{|c|}{$\mathbf{0 , 0 3}$} & \multicolumn{2}{|c|}{0,06} & \multicolumn{2}{|c|}{0,10} & \multicolumn{2}{|c|}{0,26} & \multicolumn{2}{|c|}{0,06} \\
\hline
\end{tabular}

Champ • Généralistes ayant renseigné au moins un indicateur de la ROSP, n'ayant pas l'activité trop faible ou trop forte soit 1444 médecins, France métropolitaine

Source • DREES, URPS-ML et ORS PACA, Pays de la Loire et Poitou-Charentes, panel d'observation des pratiques et des conditions d'exercice en médecine générale. 
Tableau A4.3.2 Indicateurs, prévention, régressions multiples (suite)

\begin{tabular}{|c|c|c|c|c|c|c|c|c|c|c|}
\hline & \multicolumn{4}{|c|}{ Indicateurs vaccination grippe saisonnière : \% patients } & \multicolumn{6}{|c|}{ Indicateurs iatrogénie : \% patients $>65$ ans traités par } \\
\hline & \multicolumn{2}{|c|}{$\begin{array}{c}>65 \text { ans vaccinés } \\
\text { N=1271 }\end{array}$} & \multicolumn{2}{|c|}{$\begin{array}{l}\text { 16-64 ans en ALD vaccinés } \\
\qquad \mathrm{N}=1125\end{array}$} & \multicolumn{2}{|c|}{$\begin{array}{l}\text { vasodilatateurs } \\
\qquad \mathrm{N}=1286\end{array}$} & \multicolumn{2}{|c|}{$\begin{array}{c}\text { benzodiazépines à demi- } \\
\text { vie longue } \\
\mathrm{N}=1286\end{array}$} & \multicolumn{2}{|c|}{$\begin{array}{c}\text { benzodiazépines pendant } \\
12 \text { semaines } \\
\mathrm{N}=1360\end{array}$} \\
\hline & Valeur & p-valeur & Valeur & p-valeur & Valeur & p-valeur & Valeur & p-valeur & Valeur & p-valeur \\
\hline Constante & 4,92 & $<0,01$ & $-0,80$ & 0,19 & 33,32 & $<0,01$ & $\mathbf{8 , 0 8}$ & $\mathbf{0 , 0 1}$ & 22,57 & $<0,01$ \\
\hline Zones les moins dotées & $-0,90$ & 0,07 & $-0,44$ & 0,08 & $-0,21$ & 0,68 & 1,64 & 0,19 & $-0,40$ & 0,81 \\
\hline \multicolumn{11}{|l|}{ Age } \\
\hline$<50$ ans & $-0,07$ & 0,87 & 0,34 & 0,10 & $-0,04$ & 0,87 & $-1,69$ & 0,05 & $-1,12$ & 0,32 \\
\hline$>58$ ans & $-0,75$ & 0,06 & 0,11 & 0,55 & 0,09 & 0,73 & $-1,31$ & 0,13 & $-3,38$ & $<0,01$ \\
\hline Femme & $-0,71$ & 0,06 & 0,05 & 0,77 & 0,23 & 0,12 & 0,15 & 0,85 & 1,38 & 0,18 \\
\hline Secteur 2 ou non-conventionné & $-1,78$ & $<0,01$ & 0,11 & 0,72 & 0,17 & 0,54 & 1,78 & 0,24 & 3,50 & 0,08 \\
\hline Exercice en groupe/MSP & 0,15 & 0,67 & 0,24 & 0,15 & 0,01 & 0,95 & $-0,08$ & 0,92 & 0,04 & 0,97 \\
\hline Zone rurale & $-0,21$ & 0,67 & $-0,12$ & 0,61 & $-0,68$ & 0,09 & 0,48 & 0,64 & $-1,08$ & 0,40 \\
\hline Paris et Hauts-de-Seine & $-2,65$ & $<0,01$ & $-0,09$ & 0,78 & $\mathbf{0 , 9 2}$ & $<0,01$ & $-2,75$ & 0,17 & 1,47 & 0,60 \\
\hline \multicolumn{11}{|l|}{ Niveau de vie de la commune } \\
\hline Q1 & $-0,14$ & 0,77 & 0,12 & 0,58 & $-0,48$ & 0,19 & 0,66 & 0,48 & $-0,15$ & 0,90 \\
\hline $\mathrm{Q} 4$ & $-0,17$ & 0,70 & $-0,08$ & 0,65 & $-0,01$ & 0,97 & 1,78 & 0,05 & 4,15 & $<0,01$ \\
\hline \multicolumn{11}{|l|}{ Caractéristiques de la patientèle } \\
\hline Part de patients CMU & $-0,03$ & 0,37 & $-0,01$ & 0,54 & $-0,03$ & 0,17 & $-0,13$ & 0,05 & $-0,16$ & $\mathbf{0 , 0 2}$ \\
\hline Part de $<16$ ans dans la patientèle & 0,00 & 0,94 & 0,04 & 0,08 & $\mathbf{0 , 0 7}$ & $\mathbf{0 , 0 2}$ & 0,41 & $<0,01$ & $-0,02$ & 0,84 \\
\hline Part de patients de 60 ans ou plus & $-0,01$ & 0,84 & $\mathbf{0 , 0 4}$ & $<0,01$ & 0,00 & 0,94 & 0,07 & 0,26 & $-0,21$ & $<0,01$ \\
\hline$\overline{\mathbf{R}^{2}}$ & \multicolumn{2}{|c|}{$\mathbf{0 , 0 3}$} & \multicolumn{2}{|c|}{$\mathbf{0 , 0 3}$} & \multicolumn{2}{|c|}{$\mathbf{0 , 0 3}$} & \multicolumn{2}{|c|}{$\mathbf{0 , 0 6}$} & \multicolumn{2}{|c|}{0,06} \\
\hline
\end{tabular}

Champ • Généralistes ayant renseigné au moins un indicateur de la ROSP, n'ayant pas l'activité trop faible ou trop forte soit 1444 médecins, France métropolitaine.

Source • DREES, URPS-ML et ORS PACA, Pays de la Loire et Poitou-Charentes, panel d'observation des pratiques et des conditions d'exercice en médecine générale. 